\title{
Musiktypologien und Delinquenz im Jugendalter
}

\section{Von Andreas Pöge}

Zusammenfassung: Jugendliche und ihre Lebensweisen sind seit jeher präsent in der öffentlichen Wahrnehmung und wissenschaftlichen Forschung - unter anderem, weil sie die Zukunft unserer Gesellschaft darstellen. Das Interesse gilt dabei auch und in besonderem Maße den Verhaltensweisen Jugendlicher, die normabweichend und normverletzend sind. So sorgt das Thema Jugendkriminalität immer wiederkehrend für kontroverse öffentliche Diskussionen. Dabei werden einzelnen Jugendgruppierungen - beispielsweise Sprayern, Rockern, Punks oder Skinheads - typische abweichende Verhaltensweisen zugeschrieben. Solche Gruppierungen sind oftmals mit einem speziellen und prägenden Musikstil verbunden. Mit der vorliegenden Arbeit soll anhand von Schülerbefragungsdaten aus Münster und Duisburg aufgezeigt werden, (1) welche Musikgruppierungen grundsätzlich bei Jugendlichen existieren, (2) ob kriminelles Verhalten tatsächlich in bestimmten Musiktypologien über- oder unterdurchschnittlich oft auftritt und (3) ob bestimmte Formen der Kriminalität mit bestimmten musikalischen Präferenzen einhergehen.

\section{Jugendliche Vergemeinschaftungsformen}

Mit Jugendlichen werden im Allgemeinen diejenigen Personen bezeichnet, die sich in der Altersspanne zwischen Kindheit und Erwachsensein befinden. Im Zuge der Ausweitung der Ausbildungszeiten und des immer späteren Eintritts in das Berufsleben werden in der heutigen Forschung auch die vormals ,jungen Erwachsenen“ im Alter von 20 bis 30 Jahren (Phase der Postadoleszenz) hinzugezählt, so dass die Jugendphase heutzutage ungefähr den Zeitraum zwischen dem 12. und 30. Lebensjahr umfasst. „Jugend“ kann dabei nicht nur als Durchgangsphase betrachtet werden, sondern als Kulturphänomen mit besonderen Inhalten und Lebensvollzugsformen (vgl. Hitzler / Bucher et al. 2005: 13). In dieser Lebensphase sind aus gesellschaftlicher Sicht spezielle Formen der Vergemeinschaftung zu beobachten, die nicht hinreichend mit Hilfe herkömmlicher vertikaler Ungleichheitsdimensionen (materielle Ressourcen, Berufsprestige etc.) beschrieben werden können. Zur Beschreibung dieser speziellen Vergemeinschaftungsformen kann der Begriff der Szene verwendet werden: „Szenen [...] sollen heißen: Thematisch fokussierte kulturelle Netzwerke von Personen, die bestimmte materiale und / oder mentale Formen der kollektiven Selbststilisierung teilen und Gemeinsamkeiten an typischen Orten und zu typischen Zeiten interaktiv stabilisieren und weiterentwickeln“ (Hitzler / Bucher et al. 2005: 20). Weitere Ansätze verwenden die Begriffe „Jugendkulturen“ (vgl. Baacke 2004) oder ,jugendliche Lebenswelten“ (vgl. Kleinen 2007). Bleibt man bei den „Szenen“, so sind diese gesellschaftlichen Gebilde Gesinnungsgemeinschaften von Personen, die sich meist um ein Thema (Musik, Hobby, Politik etc.) „bilden“ und als kommunikative und interaktive Gesellungsformen zumeist über die kollektive (Selbst-)Stilisierung nach außen vermittelt werden. Szenen lassen sich somit durch den Lebensstil ihrer Mitglieder unterscheiden, womit in diesem Fall das „Gesamtarrangement" des Lebensvollzuges gemeint ist. Augenfälligste Zeichen eines spezifischen jugendlichen Lebensstils - und damit Merkmale der Zugehörigkeit zu bestimmten Szenen - sind der Kleidungsstil und der Musikgeschmack. Auch spezifische Verhaltensmuster können Elemente eines Lebensstils sein, wobei Jugendliche generell häufiger zu Problem- und Risikoverhalten neigen als die Erwachsenenbevölkerung. Dies kann gesundheitlich relevantes aber auch deviantes und delinquentes Risikoverhalten sein (beispielsweise U-Bahn-Surfen, Graffiti-Sprayen in der Hip-Hop-Szene, Marihuanakonsum in der Reggae-Szene etc.; vgl. ausführlich Raithel 2004).

Insbesondere Musikgeschmack und (passender) Kleidungsstil dienen als Kommunikationsinstrumente nach innen und außen, wobei allerdings beim Kleidungsstil die eindeutige Zu- 
ordnung zu bestimmten Szenen schwieriger geworden ist. Zum Teil herrschen in unterschiedlichen Szenen gleiche Stile vor, zum Teil unterscheiden sich diese in für Außenstehende kaum erkennbaren Details. In Bezug auf den Musikgeschmack kann davon ausgegangen werden, dass vor allem im Jugendalter ein verstärktes Bedürfnis nach Musik besteht, welches durch einen hohen - in der Jugend als legitim angesehenen - Zeitaufwand befriedigt wird. In fast allen Jugendstudien findet sich das Musikhören als sehr häufige oder sogar häufigste Freizeitbeschäftigung (vgl. exemplarisch Stadt Münster 2001: 29ff., 86; Deutsche Shell 2000: 206; 2006: 77ff.; 2010: 96ff.). Dabei spielt Musik in allen Medienbereichen eine Rolle: Musik aus dem Radio, Musik im Fernsehen, heutzutage aber auch und besonders Musik in und aus dem Internet.

Über die gemeinsame Bevorzugung von Stilelementen der Rock- und Popkultur wird die Verbundenheit zu einer oder mehreren Szenen oder Jugendkulturen abgesichert (vgl. Baacke 2004: 40), in denen auch spezielle (non-)konforme Verhaltensmuster üblich sein können. Dabei nutzen Jugendliche Musik mit allen Szeneaccessoires, um etwas von ihrer eigenen Identität auszudrücken - auch oder gerade in Abgrenzung zu ihren Eltern (vgl. Behne 2007: 437). „Musik stellt eine Art Hilfsmittel zur Aufkündigung sozialer Beziehungen (Rückzug, Abkopplung) wie zu ihrer Etablierung (Abgrenzung, Distanz, Vergemeinschaftung) dar.“ (Schmidbauer / Löhr 1999: 341) Musikgeschmack ist also ein enorm wichtiger Bestandteil der jugendlichen (Selbst-)Inszenierung und des Alltags (siehe im Überblick Ferchhoff / Sander et al. 1995; Baacke 1997) und findet seinen Ausdruck eben auch in der Zugehörigkeit zu bestimmten musikalischen Gruppierungen. So sind die quantitativ größten Jugendszenen als Musikszenen konstituiert (Techno-Szene, Hip-Hop-Szene, Punk-Szene etc.). ${ }^{1}$ Innerhalb der Szenen wird der Musikgeschmack der Mitglieder dabei nicht nur auf Übereinstimmung geprüft, sondern dient als Indikator für weitere Persönlichkeitseigenschaften und nach außen als Abgrenzungsgegenstand gegen andere (vgl. Neuhoff / Motte-Haber 2007: 402).

Auch im Zusammenhang mit Konzepten der neueren Sozialstrukturanalyse lässt sich feststellen, dass Musikgeschmack in postindustriellen Gesellschaften generell mehr Bedeutung gewonnen hat und nicht nur im Umfeld von Jugendkulturen ein wichtiges Thema ist. Als erster hat Bourdieu (1987) verdeutlicht, wie kulturelle Aktivitäten (Musikgeschmack, Theaterbesuch und andere) Ausdruck der Sozialisation sein können und wie diese kulturellen Kompetenzen als Kapital in den Statuserwerb einfließen können. Innerhalb der Lebensstilkonzepte kommt dem Musikgeschmack besondere Bedeutung zu und spielt beispielsweise in den Arbeiten von Schulze (1992) und Spellerberg (1996) eine bedeutsame Rolle. Die Ausbildung von musikalischen Präferenzen wird dabei einerseits im Allgemeinen dem Bereich des wahlfreien Handelns zugeordnet, andererseits unterliegt sie sehr wohl strukturierter sozialer Ungleichheit. Beispielsweise rekrutieren sich Besucher klassischer ,,hochkultureller“"Konzerte überwiegend aus den bildungs- und statushohen Gruppen, Besucher von Konzerten mit volkstümlicher Musik und Schlager dagegen aus bildungs- und statusniedrigen Gruppen (vgl. Neuhoff 2007: 482 f.; Neuhoff / Motte-Haber 2007: 390). Als bedingende Variablen für den Musikgeschmack und das musikalische Urteilsvermögen haben sich das Elternhaus, die Schule, die Clique, die Peer-Kultur, die Medien sowie das Alter und das Geschlecht erwiesen. Auch die ethnische Herkunft hat einen Einfluss auf musikalische Sozialisation. Es konnte beobachtet werden, dass Schüler in multikulturell besetzten Klassen besonders zur gegenseitigen Abgrenzung durch

1 In einer Untersuchung aus dem Jahre 1999 konnten folgende Szenen ausgemacht werden, bei denen die Autoren allerdings selbst mittlerweile von einem großen Wandel ausgehen: Techno-Szene, Hardcore-Szene, Schwarze Szene, Skater-Szene, Graffiti-Szene, Konsolenspieler, Daily Soap-Fans, Türkische Street Gangs, Antifa-Szene und Drogen-Szene. Daneben sprechen sie von einem Jugendverband der Deutschen Lebens-Rettungs-Gesellschaft und von der Szene der Sportkletterer als Übergangsgruppe zwischen Szene und Verein (vgl. Hitzler / Bucher et al. 2005: 19ff,; siehe auch das InternetPortal www.jugendszenen.com). 
ihre musikalischen Vorlieben neigen (vgl. Neuhoff / Motte-Haber 2007: 391, 407; siehe auch Greve 2007). Hinsichtlich der Hörpräferenzen und der Sozialstruktur ist insbesondere im Jugendalter immer wieder eine positivere Bewertung ,weicherer, gefühlsbetonter“ Stile (zum Beispiel moderner Soul) durch Frauen und „härterer, aggressiverer“ Stile (beispielweise Rock oder Heavy Metal) durch Männer beobachtet worden (vgl. im Überblick Gembris 2005: 316ff.; Neuhoff / Motte-Haber 2007: 406). Dabei sind in bildungsniedrigeren Lebensstilgruppen geschlechtsspezifische kulturelle Typisierungen stärker ausgeprägt als in bildungshohen Stilen (vgl. Spellerberg 1996: 188). In den USA sind im Zusammenhang mit der Diskussion um Hochkultur und soziale Schicht empirische Befunde veröffentlicht worden, wonach Personen aus höheren Lagen nicht mehr nur klassische Musik präferieren, sondern ein größeres Spektrum an Musikarten schätzen. Unter den Musikhörern ist dabei ein statushoher Typus gehäuft aufgetreten, der klassische Musik, Rock, Pop, Jazz, Hip-Hop und andere Stile gleichermaßen bevorzugt und als ,musikalischer Allesfresser“2 bezeichnet wurde (vgl. Peterson / Kern 1996). Diesbezüglich kann also ein Zusammenhang zwischen „musikalischer Toleranz“ - ausgedrückt durch die Anzahl an positiv bewerteten Genres - und sozialer Schichtung angenommen werden. Allerdings muss man vermuten, dass die Ergebnisse nicht direkt auf Deutschland übertragbar sind (vgl. Behne 2007: 434).

\section{Jugendkriminalität und Musikgeschmack}

Während in der Wahrnehmung der Öffentlichkeit Jugendkriminalität seit Beginn der 1990er Jahre kontinuierlich und stark angestiegen ist, finden sich derlei Ergebnisse weder durchgehend in der polizeilich und justiziell registrierten Kriminalität des Hellfeldes noch im Dunkelfeld. Schon im Hellfeld kann eine stabile generelle Zunahme der Kriminalität bei Jugendlichen und Heranwachsenden nicht belegt werden (siehe Boers / Walburg et al. 2006: 64). Für das (vermeintliche) Anwachsen dieser Kriminalität zeichnet hauptsächlich eine Zunahme der Tatverdächtigenbelastungszahlen (TVBZ) in der Polizeilichen Kriminalstatistik (PKS) unter Jugendlichen und Heranwachsenden bei der ,gefährlichen“ und „schweren Körperverletzung“ zwischen 1994 und 2004 verantwortlich. ${ }^{3}$ Der Bundesverband der Unfallkassen geht indes auf Grundlage seiner Unfallversicherungsstatistiken von einem Rückgang physischer schulischer Gewalt und keiner zunehmenden Brutalisierung zwischen 1993 und 2003 aus, vielmehr von einer überdramatisierten Darstellung in den öffentlichen Medien (siehe Bundesverband der Unfallkassen 2005: 4, 21). Auch neuere Regierungsberichte gehen von einer wesentlich weniger dramatischen Situation aus, als es die immer wiederkehrenden politischen (Wahlkampf-)Debatten vermuten lassen: Die Bund-Länder-AG konstatiert, dass auf Grundlage der PKS weder gesicherte Aussagen über einen Anstieg der Jugendkriminalität noch über eine zunehmende Schwere möglich seien - im Übrigen auch nicht über einen etwaigen Migrationshintergrund der Täter (vgl. Bund-Länder-AG 2007: 5). Im Zweiten Periodischen Sicherheitsbericht der Bundesregierung wird zum Thema generalisierend festgestellt, dass Gewaltkriminalität ein qualitatives, aber kein quantitatives Problem sei (vgl. BMI / BMJ 2006: 10). Auch in den Polizeilichen Kriminalstatistiken 2008 und 2009 wird von einer Beruhigung der Lage ausgegangen (vgl. BKA 2009: 98; 2010: 98).

2 Der ungewöhnliche Name ist eine wörtliche Übersetzung des englischen Begriffes „omnivore“, der im Zusammenhang mit dieser These geläufig ist.

3 Zur Diskussion in den 1990er Jahren siehe Pfeiffer / Ohlemacher (1995); Pfeiffer / Delzer et al. (1998) und kritisch dazu Walter (1996). 
Nahezu alle Dunkelfeldbefragungen ${ }^{4}$ Jugendlicher zeigen nach einer Zunahme an Gewaltund Eigentumsdelikten zu Beginn der 1990er Jahre (vgl. Mansel / Hurrelmann 1998; Tillmann / Holler-Nowitzki et al. 1999; Lösel / Bliesener et al. 1999) seit Mitte der 1990er Jahre einen Rückgang insbesondere der Gewaltkriminalität. So etwa bei den Befragungen von Wilmers / Enzmann et al. (2002) in Hamburg, Leipzig, München und Hannover, den Schulbefragungen von Fuchs / Lamnek et al. (2005) in Bayern sowie Umfragen in Greifswald (vgl. Dünkel / Gebauer et al. 2005; 2007). Auch die Ergebnisse der Wiederholungsbefragungen durch das Kriminologische Forschungsinstitut Niedersachsen (KFN) in München, Stuttgart, Hannover und Schwäbisch Gmünd und die Schülerbefragungen in Münster und Duisburg, aus denen auch die dieser Arbeit zugrundeliegenden Daten stammen, zeigen dieselbe Befundlage (vgl. Baier / Windzio 2008; Boers / Walburg et al. 2006; Boers / Walburg 2007; zusammenfassend zum Forschungsstand siehe Heinz 2006; Steffen 2007). Neben einem Rückgang sind die Ergebnisse durch eine weitverbreitete Bagatellhaftigkeit der Jugendkriminalität und dem Phänomen der spontanen Bewährung, das heißt weitestgehender Abbruch der Tatbegehungen ohne formelle Kontrollintervention, gekennzeichnet. In den Daten aus Münster und Duisburg zeigt sich der Scheitelpunkt der Altersprävalenzkurve, also das Alter der Jugendlichen mit der höchsten Kriminalitätsbelastung, bei 14 bzw. 15 Jahren und damit überraschend früh (siehe Boers / Walburg et al. 2006: 72 f.; Boers / Reinecke et al. 2010: 58ff.).

Unterschiede zwischen den Ergebnissen der Daten aus Hell- und Dunkelfeld lassen sich in erheblichem Maße durch die Beschränkungen der offiziellen Hellfeldstatistiken erklären. Es kann nicht von einer feststehenden Relation zwischen tatsächlich begangenen und statistisch erfassten Straftaten ausgegangen werden. Laut Bundeskriminalamt (2010: 8) können sich neben tatsächlich stattgefundener Kriminalitätsänderung folgende Einflussfaktoren auf die Entwicklung der Zahlen in der Polizeilichen Kriminalstatistik auswirken: Anzeigeverhalten, polizeiliche Kontrolle, statistische Erfassung und Änderung des Strafrechts.

Durch Musik geprägten Jugendszenen wird ein großer Anteil an der jugendlichen Sozialisation zugeschrieben (vgl. Rössler / Scharfenberg 2004: 516), weshalb auch davon ausgegangen werden kann, dass sie im Hinblick auf das Erlernen antisozialer, devianter und delinquenter Verhaltensweisen wirksam sind. Zunächst kommt Musik bei der Vermittlung von (geschlechtsspezifischen) sozialen Leitbildern und Verhaltensweisen und den mit Musik verbundenen Medien eine besondere Bedeutung zu. Insbesondere in Videoclips werden oftmals ausgeprägte geschlechtsrollenstereotype und sexistische Darstellungen gezeigt, in Texten populärer Musikstücke oftmals Drogen- bzw. Alkoholmissbrauch verherrlicht (vgl. Primack / Dalton et al. 2008). Es ist wahrscheinlich, dass der Konsum solcher Clips und Songs Akzeptanzund Nachahmungseffekte zeitigt (vgl. Neuhoff / Motte-Haber 2007: 407). Studien zum Musikgeschmack haben sich seit der Vergangenheit - vorwiegend in den USA - auch oftmals mit den vermuteten negativen Auswirkungen bestimmter Musikgenres beschäftigt. Insbesondere Heavy-Metal, Rap, aber auch Rockmusik, Punk, Country sowie Oper und ihre Zusammenhänge mit Selbstmord, Drogenkonsum, Satanismus, Gewaltakzeptanz, Aggressivität, Ärger, Aufsässigkeit, abweichendem Sexualverhalten, Depression, Frauenfeindlichkeit, Hoffnungslosigkeit, antisozialem Verhalten, sozialem Misstrauen und Kriminalität waren Gegenstand der Forschung (vgl. Behne 2007: 423). Die Befundlage kann indessen kaum einheitlich oder stabil genannt werden.

4 Natürlich muss bei der Analyse der selbstberichteten Kriminalität im Auge behalten werden, dass möglicherweise nicht immer ehrlich auf die diesbezüglichen Fragen geantwortet wird. Köllisch / Oberwittler stellten allerdings fest, dass bei Befragungen die überwiegende Mehrheit der Probanden ehrlich antwortet. Zu den Methodeneffekten bei der Messung selbstberichteter Delinquenz siehe exemplarisch Köllisch / Oberwittler (2004). 
In einigen zumeist älteren Untersuchungen konnten Zusammenhänge zwischen niedrigerer Schulbildung, vermehrten Schulproblemen, verstärktem Alkohol- und Drogenmissbrauch und höheren Arrestraten (vgl. Took / Weiss 1994), insgesamt schlechten schulischen Leistungen (vgl. Roe 1992, 1995) und Selbstmordgedanken bzw. Depressionen (vgl. Martin / Clarke et al. 1993) bei Hinwendung zu Heavy Metal nachgewiesen werden. Daneben zeigten gute Schülerinnen und Schüler ein höheres Interesse an den Musikrichtungen Klassik, Jazz und Blues. In neueren Untersuchungen konnten jedoch beispielsweise die Zusammenhänge zwischen Heavy-Metal-Musik und Delinquenz (vgl. Gardstrom 1999) oder suizidalen Neigungen (Lacourse / Claes et al. 2001) nicht bestätigt werden. In jedem Fall kann allerdings wohl angenommen werden, dass nicht das Hören bzw. Präferieren von Heavy-Metal-Musik der alleinige Auslöser der genannten Probleme bzw. devianten und delinquenten Verhaltensweisen ist (vgl. Gembris 2005: 324).

In Europa konnte im Hinblick auf Drogenkonsum anhand einer groß angelegten schulbasierten Befragung in Oslo gezeigt werden, dass zwar eine musikalische Vorliebe für House und Techno mit der Einnahme von Ecstacy und Amphetaminen einhergeht, aber ein ursächlicher Zusammenhang nicht nachzuweisen ist (vgl. Pederson / Skrondal 1999). Auch eine schottische Schülerumfrage zeigt ein gemeinsames Auftreten von Ecstacy-Konsum und einer Präferenz für den Musikstil Rave. Die Musik als Auslöser einzuordnen gelingt jedoch ebenfalls nicht (vgl. Forsyth / Barnard et al. 1997).

Einen Zusammenhang zwischen Rap-Musik, Gewalteinstellungen und der Akzeptanz von Gewalthandeln fand sich in einer Untersuchung unter afro-amerikanischen Jugendlichen. Diese zeigten nach rund halbstündigem Konsum von gewalthaltigen Rap-Videos erhöhte Gewaltakzeptanz und eine erhöhte Wahrscheinlichkeit eigenen Gewalthandelns und insbesondere erhöhte Gewaltakzeptanz gegenüber Frauen (Johnson / Jackson et al. 1995). Ein solcher Übernahmeeffekt konnte in einer ähnlichen Untersuchung sogar bei Konsum von nicht gewalthaltigen, sondern solchen Musik-Videos aufgezeigt werden, in denen Frauen als sexuell untergeordnet dargestellt wurden - ein allgemein übliches Stilmittel in Musikvideos (Johnson / Adams et al. 1995). Eine Untersuchung mit Hilfe von Filmen zeigte einen Zusammenhang von sexuell aggressivem Verhalten und frauenfeindlicher Rap-Musik (Barongan / Nagayama 1995). In einer aktuellen Studie konnte nachgewiesen werden, dass generell das Hören frauenfeindlicher Songtexte bei Männern und das Hören männerfeindlicher Texte bei Frauen zu negativeren Einschätzungen und höheren Rachegefühlen gegenüber Frauen bzw. aggressiveren Antworten in Bezug auf Männer führte (vgl. Fischer / Greitemeyer 2006). Neuere Experimente zeigen darüber hinaus, dass Studenten nach dem Hören gewalthaltiger Songtexte unterschiedlicher Genres feindseligere Gefühle und aggressivere Gedanken hatten als solche, die ähnliche Musikstücke mit nicht-gewalthaltigen Texten vorgespielt bekamen (vgl. Anderson / Carnagey et al. 2003). Eine Studie unter franko-kanadischen Jugendlichen führte zum Ergebnis, dass Präferenzen für Rap-Musik signifikant aber schwach mit deviantem Verhalten zusammenhingen, wobei die Art und Weise des Zusammenhangs mit dem jeweiligen Musikgenre variierte. Vorlieben für französischen Rap wiesen den stärksten Zusammenhang auf, Vorlieben für Hip-Hop und Soul gingen mit unterdurchschnittlichen Delinquenzraten einher (vgl. Miranda / Claes 2004).

Es ist ersichtlich, dass die vorgestellten Ergebnisse generell unterschiedlich bis widersprüchlich sind. Daneben konnten kausale Erklärungszusammenhänge zwischen Musikgeschmack, -konsum und Delinquenz zum größten Teil bis heute nicht zweifelsfrei nachgewiesen werden. Fraglich bleibt insgesamt auch hier die Übertragbarkeit auf Deutschland (vgl. Behne 2007: 425). Immerhin scheinen Anhaltspunkte dafür zu bestehen, dass manche neue Musikformen (Techno, Rave) mit Drogenkonsummustern korrespondieren und dass Rap-Musik auch mit deviantem und delinquentem Verhalten zusammenhängen kann. Für darüber hinausge- 
hende kausale Erklärversuche scheint es keine hinreichenden empirischen Belege zu geben. Für diese Arbeit soll daher angenommen werden, dass spezielle Szenen - und damit zusammenhängend Lebensstile - bei Jugendlichen existieren, für die ein bestimmter Musikgeschmack typisch ist und zu denen, neben weiteren Besonderheiten, ebenfalls deviante und delinquente Verhaltensmuster gehören können. Ziel der folgenden Analyse ist es, diese Musiktypologien und ihre sozialstrukturellen Besonderheiten herauszuarbeiten und zu analysieren, ob in den zu erwartenden Musikgruppierungen bestimmte abweichende Verhaltensweisen häufiger vorkommen als in anderen.

Unter Berücksichtigung der weiteren, oben dargestellten Befunde, soll für die vorliegende Arbeit somit vermutet werden, dass

(1) ein toleranter Musikgeschmack mit einer hohen Soziallage einhergeht

(2) Vorlieben für House- und Technomusik zusammen mit hohen Alkohol- und Drogenkonsumraten auftreten.

In Bezug auf kriminelles Verhalten soll als Hypothese angenommen werden, dass sich

(3) Präferenzen für Rap-Musik zusammen mit erhöhten und

(4) Vorlieben für Klassik, Jazz und Blues mit niedrigen Delinquenzraten zeigen.

\section{Datengrundlage und Auswertungsstrategie}

Die Daten für die hier vorgestellte Analyse stammen aus der von der DFG geförderten Panelstudie „Kriminalität in der modernen Stadt“ ${ }^{5}$ bei der in den Jahren 2000 bis 2003 in einjährigen Abständen an Münsteraner und in den Jahren 2002 bis 2005 an Duisburger Schulen im Klassenverband Fragebogeninterviews durchgeführt wurden und die in Duisburg als postalische Befragung weitergeführt wird. ${ }^{6}$ Hier sollen als Grundlage drei Querschnittsdatensätze aus Münster und Duisburg dienen, die Jugendliche der zehnten Jahrgangsstufe umfassen. Dies sind die Münsteraner Daten des Jahres 2003 sowie die Duisburger Daten der Jahre 2003 und 2005. Sie ermöglichen einen Vergleich Jugendlicher derselben Jahrgangsstufe zwischen den Erhebungsorten im Jahr 2003 und einen Duisburger Trendvergleich zwischen 2003 und 2005.

5 Projektleitung: Prof. Dr. Klaus Boers, Institut für Kriminalwissenschaften, Westfälische WilhelmsUniversität Münster und Prof. Dr. Jost Reinecke, Fakultät für Soziologie, Universität Bielefeld.

6 Eine detaillierte Darstellung der einzelnen Erhebungen findet sich in den einzelnen Berichten der Methodenreihe (siehe http://www.uni-bielefeld.de/soz/krimstadt/index.html/; Pöge / Wittenberg 2007; zu Ergebnissen siehe Boers / Reinecke 2007). 
Tabelle 1: Beschreibung der Datengrundlage des zehnten Jahrgangs in Münster 2003 $(N=1.819)$, Duisburg 2003 (N=2.427) und Duisburg 2005 (N=3.243)

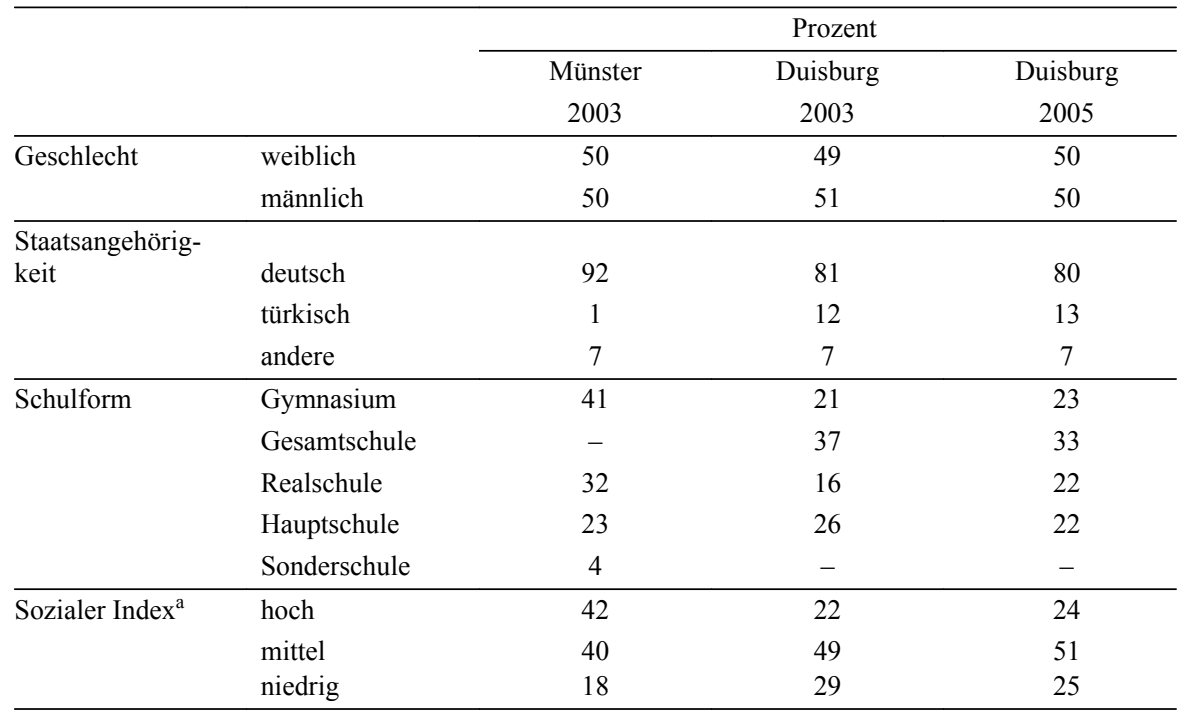

$\overline{{ }^{a}}$ Der Soziale Index setzt sich zusammen aus „Anzahl der Bücher zuhause“, „Art der Wohnung“, „Selbsteinschätzung arm / reich“.

Die absolute Fallzahl liegt in Münster bei 1.819, in Duisburg 2003 bei 2.427 und in Duisburg 2005 bei 3.243 gültigen Fällen. Die Rücklaufquoten sind mit $88 \%$, 89\% und 92\% durchweg als gut zu bezeichnen; das Durchschnittsalter der befragten Schülerinnen und Schüler beträgt 16 Jahre. In Bezug auf die Verteilung der Stichproben nach Geschlecht und Schulform (siehe Tabelle 1) liegt keine bedeutende Verzerrung im Vergleich mit der Grundgesamtheit vor, so dass eine Repräsentativität auf Stadtebene angenommen werden kann (zu den drei Datensätzen siehe auch die detaillierten Methodendokumentationen Wittenberg 2004; Brondies 2004; Kunadt 2006).

Während die Verteilung nach Geschlecht in allen drei Datensätzen nahezu gleich ist, unterscheidet sich die Verteilung nach Schulform zwischen Münster und Duisburg in Bezug auf die Gesamtschule und im Bereich der Sonder- und Hauptschulen. Auch im Bereich der Staatsangehörigkeit und des höheren sozialen Indexes in Münster (gebildet aus den Variablen „Anzahl der Bücher zuhause“, „Art der Wohnung“ und „Selbsteinschätzung arm / reich“) unterscheiden sich die Populationen deutlich (siehe Tabelle 1). Ein Befund, der aufgrund der unterschiedlichen Lebensbedingungen in den Erhebungsorten nicht verwundern kann. Die beiden kreisfreien nordrhein-westfälischen Großstädte Münster und Duisburg unterscheiden sich im Referenzzeitraum der Jahre 2002 bzw. $2003^{7}$ in der amtlichen Statistik sowohl in der deutlich kleineren Einwohnerzahl Münsters (Münster: 270.000, Duisburg: 500.000), dem deutlich höheren Anteil ausländischer Mitbürger in Duisburg (gesamt NRW 11\%, Münster 8\%, Duisburg 17\%) und der deutlich niedrigeren Arbeitslosenquote in Münster (gesamt NRW 11\%, Münster $9 \%$, Duisburg 14\%).

7 Amtliche Daten für das Jahr 2004 bzw. 2005 werden nicht gesondert ausgewiesen, da die Unterschiede in den vorgestellten Zahlen nicht relevant sind. 
Auf Grundlage der vorgestellten Datensätze sollen zunächst das kriminelle Verhalten anhand von Täteranteilen (Kriminalitätsraten) und der Musikgeschmack der Jugendlichen dargestellt werden. Auf Basis der Musikpräferenzen werden die Populationen dann mit Hilfe von latenten Klassenanalysen in Gruppen bzw. Typologien aufgeteilt, die mit weiteren Merkmalen beschrieben und im Hinblick auf spezielle Auffälligkeiten in der Delinquenzbelastung analysiert werden. Hierfür stehen in den Datensätzen für die Primäranalysen Informationen über die selbstberichtete 12-Monats-Prävalenz bei 16 Einzeldelikten bzw. -handlungen und die fünfstufige Bewertung von 15 bzw. 17 verschiedenen Musikrichtungen zur Verfügung. Die weitere Beschreibung der Typologien erfolgt neben den in Tabelle 1 dargestellten Merkmalen über Werthaltungen, Freizeitverhalten, Film- und Fernsehpräferenzen sowie dem selbstberichteten Alkohol- und Drogenkonsum der Jugendlichen (zu diesen beschreibenden Merkmalen vgl. genauer Pöge 2002; Boers / Pöge 2003; Pöge 2007 b). Diese exploratorische Vorgehensweise soll dazu dienen, Zusammenhänge zwischen den genannten Merkmalen aufzudecken. In dem oben geschilderten Sinne ist das Ziel, festzustellen, in welchen Musiktypologien möglicherweise bestimmte abweichende Verhaltensweisen häufiger oder seltener vorkommen als in anderen. Die hier vorliegende Analyse kann jedoch nicht im Sinne eines Erklärungsansatzes von Kriminalität verstanden werden. Allein die Zughörigkeit zu bestimmten Musikgruppierungen wird kriminelles Verhalten nicht befriedigend erklären können, obwohl bestimmte (non-)konforme Verhaltensweisen durchaus Elemente bestimmter, durch Musik geprägter Lebensstile sein können.

\section{Selbstberichtete Kriminalität}

Betrachtet man die Delinquenzbelastung der befragten Jugendlichen, so ist zunächst festzustellen, dass der Anteil derjenigen, die in den letzten zwölf Monaten irgendeines der abgefragten Delikte begangen hatten (Gesamtprävalenz), in Münster 2003 und Duisburg 2005 bei ungefähr einem Drittel liegt, in Duisburg 2003 dagegen etwas höher (siehe Tabelle 2 und vgl. dazu Boers / Walburg et al. 2006; Boers / Walburg 2007). ${ }^{8}$ Man kann dies mit aller gebotenen Vorsicht als Rückgang der Jugendkriminalität in Duisburg zwischen den Jahren 2003 und 2005 interpretieren. Dieser Rückgang findet sich auch in den Prävalenzraten aller dargestellten Deliktsgruppen und Einzeldelikte. Darüber hinaus sind mit Ausnahme der Gewaltdelikte die meisten der ausgewiesenen Einzeldeliktsraten in Duisburg 2005 ebenfalls niedriger als in Münster 2003. Bei den Gruppen der Gewalt-, Eigentums- und Sachbeschädigungsdelikte finden sich sowohl in Münster als auch in Duisburg die größten Täteranteile im Bereich der Eigentumskriminalität. Häufigstes Eigentumsdelikt ist hierbei in beiden Städten Ladendiebstahl. Daneben berichten die Jugendlichen in Münster auffällig oft von einem Fahrraddiebstahl. Beide Erhebungsorte weisen zu beiden Zeitpunkten die klassische Sachbeschädigung (Vandalismus) als das am häufigsten begangene Sachbeschädigungsdelikt auf, wobei sich alle Einzel-Prävalenzraten in diesem Bereich auf einem ähnlichen Niveau bewegen. Gewaltdelikte sind stark dominiert von der leichtesten der abgefragten Straftaten: Körperverletzung ohne Waffen. Während die schweren Gewaltdelikte Raub, Handtaschenraub und Körperverletzung mit Waffen durchweg nur von einem sehr kleinen Teil der Befragten angegeben werden, erreicht diese durchaus zweistellige Raten. Trotz leicht rückläufiger Zahlen und vergleichbarer oder höherer Werte in anderen Städten, sind diese Belastungsziffern nicht unerheblich, zumal bei der Fragestellung darauf geachtet wurde, harmlose Raufsituationen auszuschließen.

8 Da im weiteren Verlauf der Arbeit nur mit ungewichteten Daten gerechnet wird, erfolgt hier die Darstellung der Kriminalitätsraten ebenfalls anhand der ungewichteten Daten. Aus diesem Umstand erklären sich eventuelle geringfügige Differenzen zu den Angaben in der angegebenen Literatur. 
Tabelle 2: Täteranteile in Münster und Duisburg (Jahresprävalenz, ungewichtete Daten)

\begin{tabular}{lccc}
\hline & & Prozent & \\
\cline { 2 - 4 } & Münster & Duisburg & Duisburg \\
& 2003 & 2003 & 2005 \\
\hline Drogenhandel & 7 & 7 & 5 \\
\hline Raub & 2 & 4 & 3 \\
Handtaschenraub & 0 & 1 & 1 \\
Körperverletzung mit Waffe & 1 & 4 & 2 \\
Körperverletzung ohne Waffe & 10 & 17 & 12 \\
\hline Gewaltdelikte gesamt & 11 & 19 & 14 \\
\hline Scratching & 7 & 11 & 7 \\
Sachbeschädigung & 9 & 14 & 8 \\
Graffiti & 8 & 9 & 6 \\
\hline Sachbeschädigungsdelikte gesamt & 17 & 23 & 15 \\
\hline Einbruchsdiebstahl & 3 & 3 & 2 \\
Hehlerei & 7 & 9 & 6 \\
Kfz-Diebstahl & 2 & 3 & 2 \\
Kfz-Aufbruch & 1 & 2 & 1 \\
Automatenaufbruch & 2 & 4 & 2 \\
Fahrraddiebstahl & 11 & 6 & 4 \\
Ladendiebstahl & 14 & 13 & 9 \\
sonstiger Diebstahl & 3 & 3 & 2 \\
\hline Eigentumsdelikte gesamt & 25 & 24 & 18 \\
\hline Gesamtprävalenz & 35 & 40 & 30 \\
\hline
\end{tabular}

\section{Musikstile}

Die Analyse der musikalischen Präferenzen erfolgt durch eine Hauptachsen-Faktorenanalyse (PAF) mit Varimax-Rotation aller abgefragten Musikitems. ${ }^{9}$ Für die Analysen wurden nur die durchgängig in beiden Städten und Jahren vorhandenen Items berücksichtigt. Nach der Eliminierung einiger nicht valider Items konnten in allen Datensätzen gut interpretierbare vierfaktorielle Lösungen erreicht werden, wobei die extrahierten Faktoren als Musikstile bezeichnet werden.

Als Bezeichnung des ersten Faktors, der durch Rap, Hip-Hop und Funk / Soul / R 'n' B gekennzeichnet ist, wird Black Music verwendet. Der zweite Faktor drückt mit den Einzelitems Punk, Hardrock / Metal sowie Crossover / Alternative / Independent eine Vorliebe für gitarrenlastige Musik aus und soll deshalb Rock genannt werden. Der dritte Faktor lässt auf einen anspruchsvollen, gehobenen Musikgeschmack schließen, da hier die Items Blues / Jazz sowie Klassik hoch laden und wird als Neue E-Musik interpretiert. ${ }^{10}$ Der vierte und letzte Faktor mit

9 Die Musikrichtungen waren im Einzelnen: Techno, Wave / Gothic, Hip-Hop, Punk, Klassik, Pop / Rock, Rap, House, Raggae / Ska, Hardrock / Metal, Funk / Soul / R 'n' B, Blues / Jazz, Schlager, Crossover / Alternative / Independent, Brit Pop / Hamburger Schule, Türkische Musik und Gabba / Gabber.

10 Wobei die Abkürzung „E-Musik“ gemeinhin für sogenannte „ernste“ Kunstmusik, „ernst zu nehmende“ oder „kulturell wertvolle“ Musik gebräuchlich ist. Das Adjektiv „neue“ wurde dem Begriff hinzugefügt, um ihn von der ,alten“ klassischen Musik abzugrenzen. 
den Musikrichtungen House und Techno verkörpert einen Musikgeschmack, der sich stark an Diskotheken- bzw. moderner Tanzmusik orientiert und soll aufgrund der Tanzorientierung Dance heißen.

Um deskriptive Analysen zu ermöglichen, werden Mittelwertindizes verwendet, indem die Variablen, die auf einen Faktor laden, zu einer neuen Musikstilvariablen mit drei Kategorien zusammengefasst werden. $\mathrm{Zu}$ berücksichtigen ist hierbei, dass es sich bei der Bewertung der Stile nicht um ein „Entweder oder“ handelt. Es mag Jugendliche geben, die jeden Musikstil gut oder auch schlecht finden. Daneben ist zu beachten, dass die angewendete Vorgehensweise auf Basis von Faktorenanalysen nur ,trennscharfe“ Musikstile liefert. ${ }^{11}$ Mit solch einem Vorgehen wird der Informationsgehalt einer Gruppe von Rating-Items erwünschtermaßen reduziert, was dazu führt, dass nun nicht mehr alle einzelnen Musikrichtungen dargestellt werden. Dies bedeutet natürlich nicht, dass einzelne eliminierte Musikrichtungen nicht beliebt oder bedeutsam wären, die Musikrichtung ist nur für die Dimensionalität nicht relevant.

Tabelle 3: Bewertung der Musikstile in Münster 2003 (MS03) und Duisburg 2003 sowie 2005 (DU03, DU05)

\begin{tabular}{lccc}
\hline & \multicolumn{3}{c}{ Prozent } \\
\cline { 2 - 4 } & MS03 & DU03 & DU05 \\
\hline gut & 63 & 61 & 62 \\
mittel & 10 & 11 & 8 \\
schlecht & 27 & 29 & 30 \\
\hline
\end{tabular}

(a) Black Music

\begin{tabular}{lccc}
\hline & \multicolumn{3}{c}{ Prozent } \\
\cline { 2 - 4 } & MS03 & DU03 & DU05 \\
\hline gut & 19 & 20 & 18 \\
mittel & 7 & 7 & 6 \\
schlecht & 74 & 73 & 76 \\
\hline
\end{tabular}

(c) Rock

\begin{tabular}{lccc}
\hline & \multicolumn{3}{c}{ Prozent } \\
\cline { 2 - 4 } & MS03 & DU03 & DU05 \\
\hline gut & 32 & 30 & 16 \\
mittel & 18 & 15 & 14 \\
schlecht & 50 & 55 & 70 \\
\hline
\end{tabular}

(b) Dance

\begin{tabular}{lccc}
\hline & \multicolumn{3}{c}{ Prozent } \\
\cline { 2 - 4 } & MS03 & DU03 & DU05 \\
\hline gut & 16 & 12 & 9 \\
mittel & 14 & 11 & 11 \\
schlecht & 70 & 77 & 80 \\
\hline
\end{tabular}

(d) Neue E-Musik

In allen drei Datensätzen zeigt sich die bekannte Beobachtung, dass auch unter deutschen Jugendlichen in der ersten Dekade des 21. Jahrhunderts vornehmlich die Hip-Hop-Kultur vorherrscht (siehe Tabelle 3 und vgl. Farin 2001: 132ff.). Fast zwei Drittel aller Befragten finden Black Music gut - diese Beobachtung scheint auch über den untersuchten Zeitraum stabil zu sein. Mit deutlichem Abstand rangiert der tanzorientierte Musikstil Dance auf dem zweiten Platz der Beliebtheitsskala. Hierbei ist zu bemerken, dass mit rund der Hälfte aller Befragten eine Mehrzahl diesen Musikstil bereits schlecht findet und im Duisburger Trendvergleich die Beliebtheit zwischen 2003 und 2005 rapide abzunehmen scheint. Bei dem gitarrenbetonten Musikstil Rock findet sich ein nochmals deutlich niedrigerer Zustimmungswert. Im Zeitvergleich wird darüber hinaus deutlich, dass die Zustimmung in geringem Umfang ebenfalls eher noch abnimmt. Am unbeliebtesten ist der gehobene, anspruchsvolle Musikgeschmack Neue E-Musik. Auch hier geht die Zustimmung im Trendvergleich noch weiter zurück. Die größere Beliebtheit dieses Stils in Münster könnte ein Indiz für den Umstand der geschilderten sozialen Unterschiede sein, die auch im deutlich höheren Wert des sozialen Indexes deutlich werden.

11 Ein Item wie Pop / Rock wird als nicht valide eliminiert, da es eben nicht trennscharf ist bzw. zu einem bestimmten Faktor gehört. 
Was kann man also zusammenfassend über den Musikgeschmack in Münster und Duisburg sagen? Zunächst ist festzuhalten, dass die Reihenfolge der Beliebtheit zwischen den beiden Städten und auch zwischen den Befragungsjahren gleich ist, wir also von einer übergreifend stabilen Befundlage sprechen können. Inhaltlich wird deutlich, dass Black Music, bestimmt durch Hip-Hop und Rap, der vorherrschende Musikstil der Gegenwartsmusikkultur unter Jugendlichen ist, der seine Vormachtstellung über die Jahre sogar noch ausbauen kann. Alle anderen Musikstile scheinen - bei aller interpretatorischer Vorsicht - in ihrer Beliebtheit eher zu sinken.

\section{Musiktypologien}

Das Ziel der nachfolgend vorgestellten Analysen ist, die Jugendlichen anhand ihres Musikgeschmacks zu klassifizieren, das heißt, in Gruppen mit jeweils klarem Musikgeschmacksprofil einzuteilen. Für diese Klassifikationsanalysen wird das Verfahren der latenten Klassenanalyse (LCA) auf Grundlage der validen und in drei Kategorien umkodierten Einzel-Musikitems angewendet. Die LCA geht im Standardmodell (siehe Lazarsfeld 1950; Lazarsfeld / Henry 1968) von einer Reihe manifester Variablen, deren Werte direkt beobachtbar sind (hier: Musikitems) und einer latenten Variablen, deren Werte nicht direkt beobachtbar sind, aus. Die latente Variable wird dabei anhand der Daten „konstruiert“, und ihre Kategorien werden üblicherweise als latente Klassen bezeichnet (Andreß / Hagenaars et al. 1997: 211). Sie ist ein probabilistisches Klassifizierungsverfahren, was bedeutet, dass die einzelnen Fälle nicht deterministisch einem Cluster zugeordnet, sondern die Wahrscheinlichkeiten der Zugehörigkeit zu jeder Klasse angegeben werden. Wie bei der verwandten Clusteranalyse ist allerdings auch bei der LCA das Ziel, innerhalb der Gruppen bzw. Klassen die Homogenität und zwischen den Klassen die Heterogenität zu maximieren. Für die vorliegenden Analysen wird das Software-Paket Latent GOLD verwendet (siehe Vermunt / Magidson 2005 a, b). Dieses Programm hat sich mit seinem Verfahren und den zugrunde liegenden EM- und Newton-Raphson-Algorithmen im praktischen Vergleich mit anderer Software und Verfahren für Klassifizierungsaufgaben als sehr geeignet erwiesen (vgl. Bacher / Wenzig et al. 2004: 21; Bacher / Pöge / Wenzig 2010: 452ff.). Das durchgeführte Verfahren wird als Latent Class Cluster Analysis bezeichnet. Hierbei werden als Modelle mehrere latente Klassenanalysen mit unterschiedlicher Klassenzahl gerechnet. Die Festlegung auf eine Lösung und insbesondere der Klassenzahl erfolgte in dieser Arbeit auf Grundlage inhaltlicher Überlegungen, unterstützt durch statistische Kriterien (siehe Tabelle 4). 
Tabelle 4: Zusammenfassung der Informationsmaße für die Auswahl der Klassenzahl

\begin{tabular}{|c|c|c|c|c|}
\hline Klassen & LL & BIC(LL) & p-value & Class.Err. \\
\hline \multicolumn{5}{|c|}{ Münster 2003} \\
\hline 1 & $-16893,9688$ & 33937,6137 & 0 & 0 \\
\hline$\cdots$ & $\begin{array}{c}\ldots \\
-15250,6160\end{array}$ & $\begin{array}{c}\ldots \\
31593,8678\end{array}$ & $\ldots$ & $\begin{array}{c}\ldots \\
0,1578\end{array}$ \\
\hline 8 & $-15160,5997$ & 31570,9951 & 0 & 0,1646 \\
\hline 9 & $-15106,3317$ & 31619,6190 & 0 & 0,1657 \\
\hline \multicolumn{5}{|c|}{ Duisburg 2003} \\
\hline 1 & $-22084,0775$ & 44323,6606 & 0 & 0 \\
\hline $\begin{array}{l}\ldots \\
8\end{array}$ & $-19646,5539$ & 40591,5789 & $\begin{array}{c}\ldots \\
0\end{array}$ & 0,1594 \\
\hline 9 & $-19549,2986$ & 40560,349 & 0 & 0,1724 \\
\hline 10 & $-19488,8342$ & 40602,701 & 0 & 0,1748 \\
\hline \multicolumn{5}{|c|}{ Duisburg 2005} \\
\hline 1 & $-27331,5661$ & 54824,4123 & 0 & 0 \\
\hline$\dddot{\ldots}$ & $-23935,8818$ & $\begin{array}{c}\ldots \\
49218,4528\end{array}$ & $\dddot{\ldots}$ & $0, \ldots$ \\
\hline 9 & $-23847,4939$ & 49211,0212 & 0 & 0,1844 \\
\hline 10 & $-23772,6365$ & 49230,6506 & 0 & 0,1931 \\
\hline
\end{tabular}

grau hinterlegt: ausgewählte Lösungen

Neben der Betrachtung der einzelnen Zuordnungswahrscheinlichkeiten (tabellarisch nicht dargestellt) und der Klassifikationsfehler wurde vor allem das Bayesian Information Criterion (BIC, vgl. Schwarz 1978; Davier 1997) herangezogen, wobei das Modell mit dem niedrigsten BIC-Wert am besten zu den Daten passt. Im Bereich der Modelle, in denen der BIC ein Minimum annimmt, wurde inhaltlich im Sinne einer Sensitivitätsanalyse entschieden, wie viele Klassen zur plausiblen Beschreibung der Daten benötigt werden und ab wann Redundanz auftritt (vgl. Bacher / Pöge / Wenzig 2010: 425 f.). Nach der Modellauswahl kann das Rechenverfahren erneut durchgeführt werden, wobei jeder Fall dann derjenigen Klasse „fest“ zugeordnet wird, zu der er die höchste Zuordnungswahrscheinlichkeit besitzt. So entstehen disjunkte Gruppen, die weiter ausgewertet werden können (vgl. Pöge 2007 b). ${ }^{12}$

Für die Profilbeschreibung der einzelnen Musiktypologien werden neben der Größe zunächst die Musikpräferenzen der Klassenangehörigen aufgezeigt. Als beschreibende Variablen werden dann das Geschlecht, Staatsangehörigkeit, Schulform, sozialer Index und die Werthaltungen der Befragten, deren Freizeitverhalten, Film- und Fernsehpräferenzen sowie Gruppenselbstbeschreibungen analysiert. Des Weiteren werden der selbstberichtete Alkohol- und Drogenkonsum sowie die Jahresprävalenzen aller vorgestellten Delikte (Gesamtprävalenz) und der aggregierten Gewalt-, Eigentums- und Sachbeschädigungsdelikte ausgewertet. Das ein-

12 Hier wurde als konservatives Verfahren die LCA für nominale Daten gerechnet. Design-Effekte bei der Berechnung von Standardfehlern und Signifikanztests, wie sie bei den vorliegenden Daten vermutet werden könnten, treten daher nicht auf. Latent GOLD bietet die Möglichkeit, diese Effekte zu berücksichtigen. Für den Datensatz aus Duisburg 2003 wurde dies exemplarisch durchgeführt. Da die Daten aus einer Schülerbefragung im Klassenverband stammen, können die 114 Schulklassen (Klumpeneffekt) der Stichprobe als ,Primary Sample Units“(PSU) berücksichtigt werden. Ebenfalls kontrolliert werden kann die Gesamtzahl der 196 Schulklassen in Duisburg 2003 laut Schulstatistik (vgl. Brondies 2004), die dann als „Population Size“ in Latent GOLD einfließen. Die Vergleichsrechnung ergibt exakt dasselbe Ergebnis wie die Berechnung ohne die genannten Parameter. Insbesondere die Klassenzugehörigkeit der einzelnen Fälle ändert sich nicht, so dass auf weitere Rechnung mit den übrigen Daten verzichtet wurde (vgl. Vermunt / Magidson 2005 b: $97 \mathrm{ff}$.). 
zelne Delikt „Graffiti oder Tags sprayen“ wird gesondert aufgeführt, da es als ein sehr szenespezifisches Delikt gilt und hier versucht werden soll, den Zusammenhang mit einer Musikszene aufzuzeigen. Diese deskriptiven Auswertungen basieren auf Kreuztabellen mit einer Analyse der standardisierten Residuen im Rahmen der Konfigurationsfrequenzanalyse (vgl. Krauth / Lienert 1973). Im Folgenden werden nur die Ergebnisse dargestellt, bei denen sich in den Kreuztabellen die beobachteten Zellhäufigkeiten überzufällig (auf mindestens 5-prozentigem Signifikanzniveau) von den erwarteten Häufigkeiten unterscheiden (vgl. Lautsch / Weber 1995; Bortz 1999; Bühl / Zöfel 2005).

\section{Tabelle 5: Übersicht über die Musiktypologien in Münster und Duisburg}

\begin{tabular}{lll}
\hline $\begin{array}{l}\text { Münster } \\
2003\end{array}$ & $\begin{array}{l}\text { Duisburg } \\
2003\end{array}$ & $\begin{array}{l}\text { Duisburg } \\
2005\end{array}$ \\
\hline Vielseitige (14\%) & $\begin{array}{l}\text { Vielseitige (9\%) } \\
\text { vielseitige Mädchen (8\%) }\end{array}$ & Vielseitige (5\%) \\
\hline angep. Hip-Hopper (15\%) & angep. Hip-Hopper (13\%) & angep. Hip-Hopper (17\%) \\
\hline Hip-Hopper u. Raver (16\%) & Hip-Hopper u. Raver (19\%) & Hip-Hopper u. Raver (11\%) \\
\hline reine Hip-Hopper (18\%) & reine Hip-Hopper (19\%) & reine Hip-Hopper (26\%) \\
\hline Party-Raver (8\%) & Party-Raver (10\%) & Party-Raver (5\%) \\
\hline Außenseiter (8\%) & Außenseiter (12\%) & Außenseiter (13\%) \\
\hline Rocker (8\%) & Rocker (7\%) & Rocker (11\%) \\
& & privilegierte Rocker (6\%) \\
durchschnittl. Rocker (7\%) & \\
\hline konf. Durchschnittl. (13\%) & &
\end{tabular}

Die Ergebnisse der Klassifikationsanalysen führen als beste Lösungen in Münster zu acht und in Duisburg zu jeweils neun Typologien (siehe Tabelle 4; vgl. genauer Pöge 2007 b). Insgesamt gesehen lässt sich eine große Ähnlichkeit im Typologiegefüge aller drei untersuchten Datensätze feststellen. Die Profile der meisten Typologien finden sich in beiden Städten und zu beiden Zeitpunkten (siehe Tabelle 5). An dieser Stelle kann aus Platzgründen nicht auf eine detaillierte Beschreibung jeder einzelnen Typologie eingegangen werden, sondern es soll aus vergleichender und zusammenfassender Perspektive eine Überblicksbeschreibung stattfinden (zur Vertiefung siehe Pöge 2007 a, b).

In den Tabellen 6, 7 und 8, in denen die Profile der einzelnen Typologien visualisiert werden, beziffern die Zahlangaben mit einem Pluszeichen versehen im Bereich der Musik den prozentualen Anteil der Jugendlichen einer jeweiligen Typologie, der den angegebenen Musikstil präferiert. Mit einem Minuszeichen versehen stellen sie den Anteil derjenigen dar, die den Stil ablehnen. Im Bereich der Delinquenz bedeuten die Zahlenangaben bei Pluszeichen Täteranteile und bei Minuszeichen „Nicht“-Täteranteile. ${ }^{13}$ Die ausgewiesenen Musikpräferenzen und Delinquenzbelastungszahlen der einzelnen Typologien sind mit den Durchschnittsangaben aus den Tabellen 2 und 3 zu vergleichen.

Das Profil der Vielseitigen tritt in allen drei Datensätzen auf, in Duisburg 2003 gibt es allerdings zwei vielseitige Typologien, von denen eine durch einen hohen Mädchenanteil auf-

13 In Tabelle 6 bedeutet also beispielsweise die Angabe , +93 “ in Klasse 1 bei Black Music, dass ein Anteil von 93\% der Jugendlichen dieser Typologie den Musikstil Black Music „gut findet“. Die Angabe von ,, -82 “ bei Gewaltdelinquenz in Typologie 4 bedeutet, dass ein Anteil von $82 \%$ keine Gewaltstraftat im vorhergegangenen Jahr angegeben hat. 
fällt. Diese Gruppierungen sind vor allem von einer Zustimmung zu allen Musikstilen geprägt. Im Bereich der Werthaltungen erfahren traditionelle Wertvorstellungen in Münster und Duisburg 2003 Ablehnung. Die Gruppenmitglieder sind sozial privilegiert, so berichten in Münster 2003 und Duisburg 2005 viele Jugendliche von einem hohen Sozialstatus, und der Anteil der Gymnasiasten ist (besonders in Münster) hoch. Sie bezeichnen die Mitglieder des eigenen Freundeskreises als Skater, Punks oder Heavy-Metal-Fans, in Duisburg auch als Sprayer. Die Jugendlichen dieser Gruppen spielen überdurchschnittlich häufig ein Musikinstrument, machen in einer Band Musik oder singen im Chor, gehen oft ins Kino oder Theater und malen überdurchschnittlich häufig. Des Weiteren schauen sie gerne Krimis und Science-Fiction-Filme sowie politische Magazine oder Dokumentationen (abgesehen von der „Mädchentypologie“ in Duisburg 2003). Das deviante und delinquente Verhalten in Münster 2003, Duisburg 2003 (Mädchen) und Duisburg 2005 ist durchschnittlich. In der nicht-weiblichen Klasse aus Duisburg 2003 findet sich bagatellhaftes kriminelles Verhalten (Graffiti, Sachbeschädigung). Die Größe dieser gehobenen, vielseitigen Typologien unterscheidet sich zwischen den Erhebungsorten und -zeitpunkten deutlich. Die oben geschilderte These des vielseitigen Musikgeschmacks in Kombination mit hohem Sozialstatus lässt sich mit diesen vielseitigen Typologien belegen. 


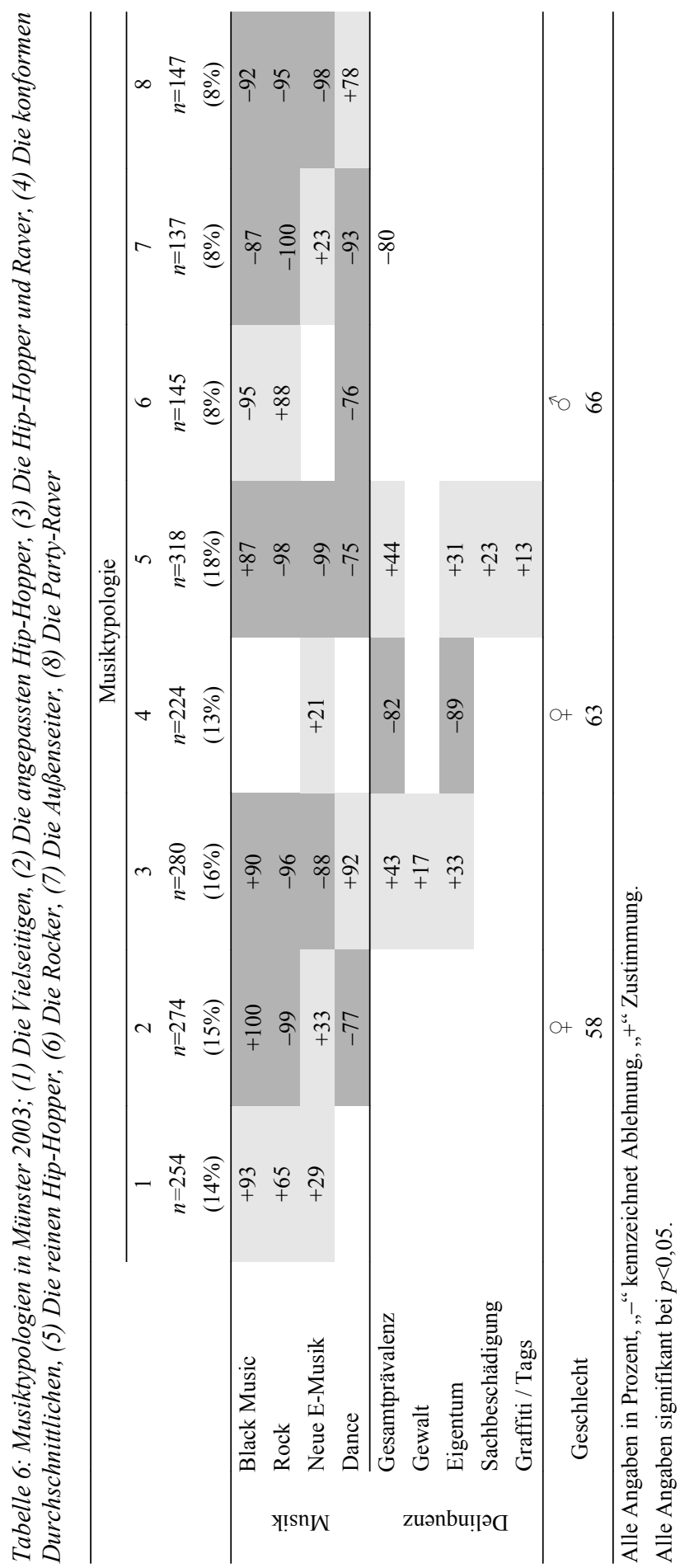




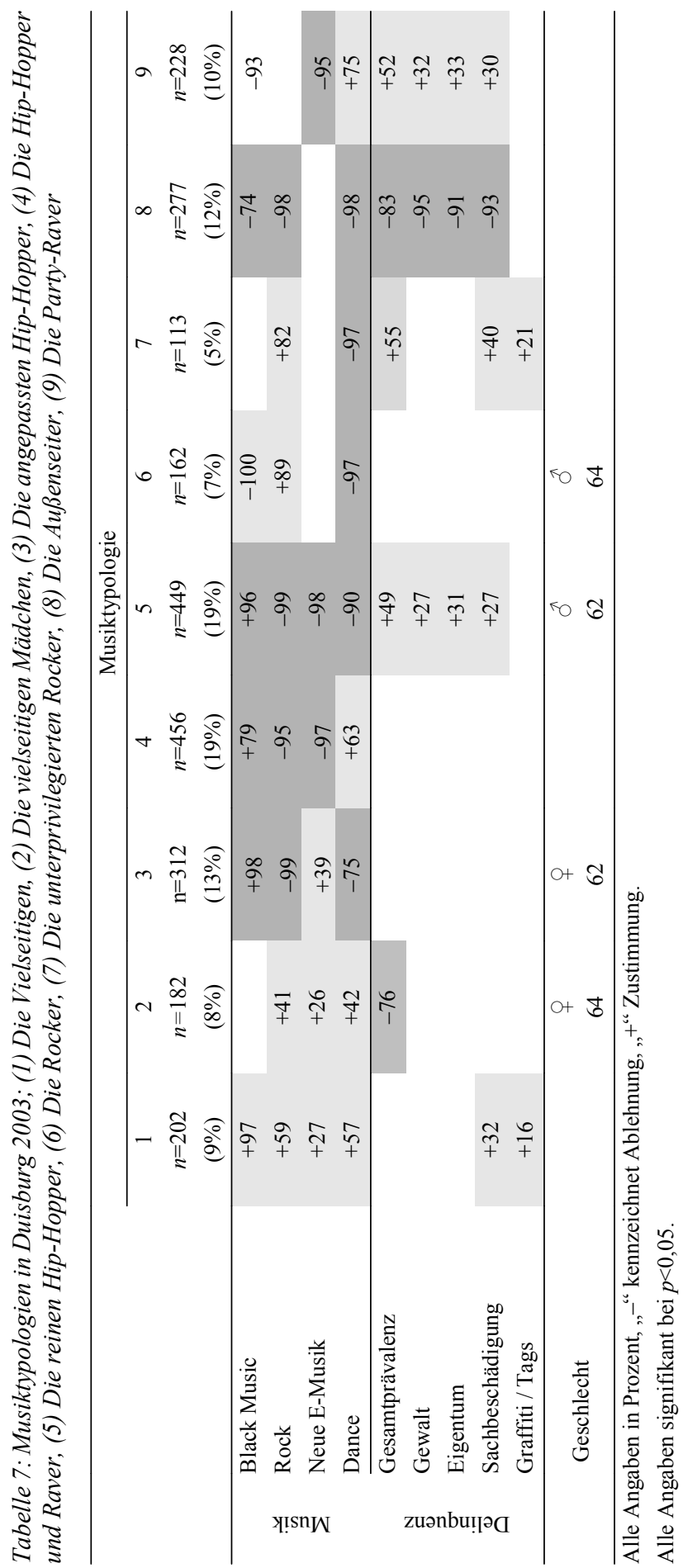




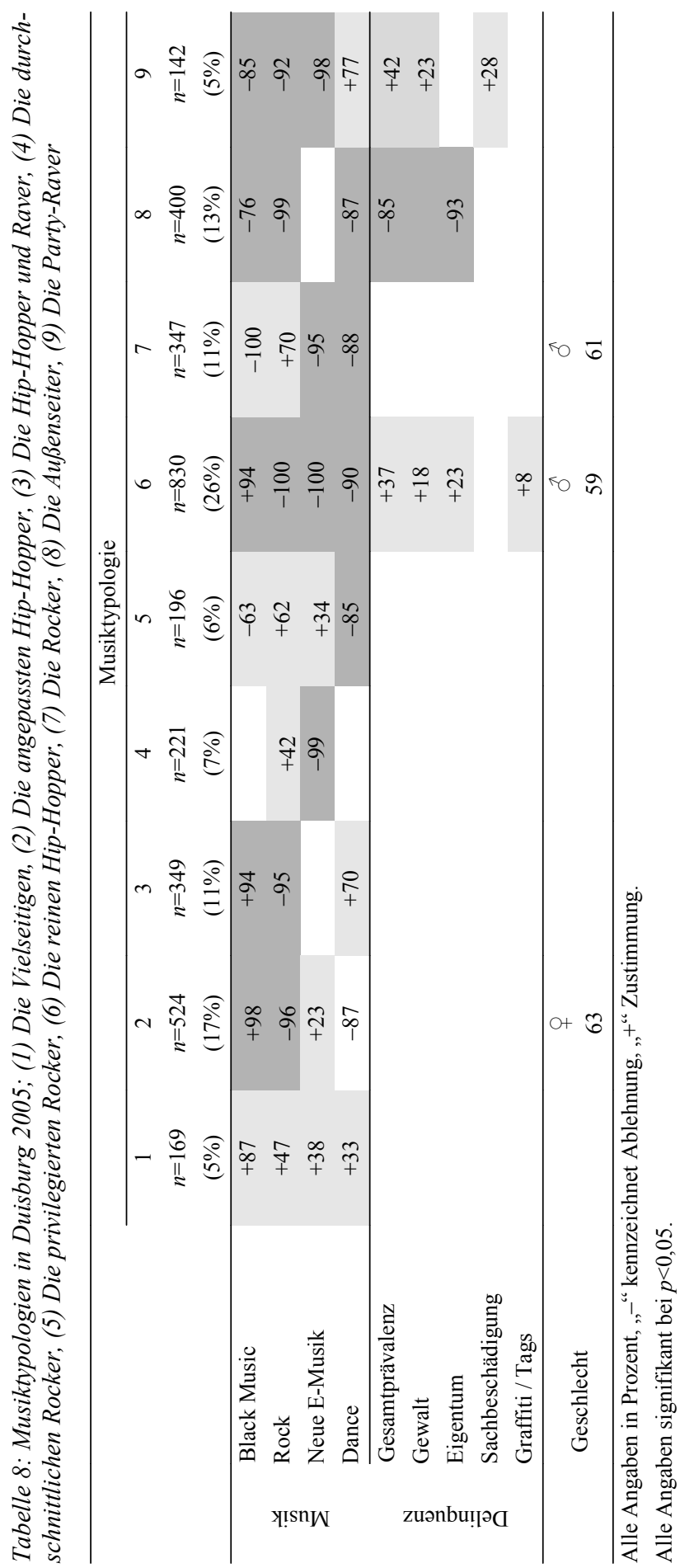


Die Musiktypologien der angepassten Hip-Hopper weisen eine überdurchschnittlich weit verbreitete Zustimmung zu Black Music und Neuer E-Musik sowie Ablehnung von Rock und Dance auf. Kennzeichnend ist ein erhöhter Frauenanteil und der Migrationshintergrund überdurchschnittlich vieler Gruppenmitglieder. Viele Jugendliche stimmen religiösen sowie (außer in Duisburg 2005) traditionellen Werthaltungen zu und bezeichnen sich dem Musikgeschmack entsprechend oft als Hip-Hopper und Rapper. Zu den beliebtesten Freizeitbeschäftigungen gehören Kinobesuche, das Treffen von Familienangehörigen und das Shoppen. Die Filmvorlieben sind eher ,typisch weiblich“ geprägt: Es finden sich unter den beliebten Genres Unterhaltungsserien, Fernsehshows und Love Stories. Die Alkoholkonsumraten sind auffallend niedrig, die Delinquenzraten durchschnittlich.

Das Profil der Hip-Hopper und Raver besteht aus der weit verbreiteten Zustimmung zu Black Music und Dance sowie der Ablehnung von Rock und (mit Ausnahme von Duisburg 2005) Neuer E-Musik. Die Jugendlichen dieser Typologien bezeichnen den eigenen Freundeskreis als Hip-Hopper und Raver bzw. Techno-Fans, in Duisburg 2003 auch als Skater. In Duisburg ist die Einstellung zu den Werthaltungen in beiden Erhebungsjahren durch eine Ablehnung von Religion geprägt, in Duisburg 2005 darüber hinaus durch die Zustimmung zu Hedonismus. In der Freizeit besuchen die Jugendlichen oft Partys, und der damit einhergehende Alkoholkonsum ist (außer in Duisburg 2003) überdurchschnittlich hoch. In Duisburg 2005 findet sich auch ein überdurchschnittlich hoher Drogenkonsum, der eigentlich generell vermutet wurde. Weitere verbreitete Freizeitbeschäftigungen sind das Shoppen und das Abhängen. Als Filmgenres sind Unterhaltungssendungen und Fernsehshows beliebt, in Duisburg auch Musikvideos (MTV oder VIVA). In diesen Typologien liegen in Münster - im Gegensatz jedoch zu Duisburg - deutlich erhöhte Kriminalitätsraten vor. Die Größe der Gruppierungen ist durchaus unterschiedlich.

Von den Jugendlichen der reinen Hip-Hop-Typologien wird einzig der Musikstil Black Music für gut befunden, alle anderen Musikstile werden abgelehnt. Der Sozialstatus der Gruppenmitglieder ist niedrig, der Anteil der Hauptschülerinnen und -schüler und derjenigen Jugendlichen mit (vorwiegend türkischem) Migrationshintergrund erhöht. In Duisburg umfasst dieser Typus mehr Jungen als Mädchen. Es werden traditionellen und (außer in Duisburg 2005) hedonistischen Werthaltungen zugestimmt, in Duisburg kommt, vermutlich wegen des hohen Migrantenanteils, eine verbreitete Religionsbefürwortung hinzu. Die Jugendlichen dieser Gruppen bezeichnen die eigene Peer-Group passend zum Musikgeschmack als Hip-Hopper und Rapper. In Bezug auf das Freizeitverhalten fallen in Duisburg überdurchschnittlich häufige Nennungen von Jugendzentrumsbesuchen und Musikmachen am eigenen PC auf. Beliebte Filmgenres sind Kung-Fu- und in Duisburg auch Action- und (mit Ausnahme von Duisburg 2005) Kampfsportfilme sowie Sportsendungen; in Duisburg 2005 auch Horrorfilme. Des Weiteren finden sich in Duisburg - möglicherweise ebenfalls wegen des hohen Anteils an türkischen Jugendlichen - niedrige Alkoholkonsumraten. Diese Musiktypologie ist die einzige, welche in Münster und Duisburg beider Jahre durch erhöhte Täterraten auffällt. Zwar sind die Gewaltdeliktsraten in Münster 2003, die Sachbeschädigungsraten in Duisburg 2005 und die Raten des Einzeldelikts Graffiti-Sprayen in Duisburg 2003 nur durchschnittlich, die sonstige datensatzübergreifend deutlich erhöhte Kriminalitätsbelastung in dieser Typologie ist indes äußerst bemerkenswert und besorgniserregend. Der vermutete Zusammenhang zwischen RapMusik und erhöhter Kriminalität zeigt sich hier ganz offenkundig.

In den Typologien der Party-Raver wird ausschließlich der Musikstil Dance gerne gehört, alle anderen Stile (mit Ausnahme von Rock in Duisburg 2003) werden weit verbreitet abgelehnt. In diesen Gruppen befinden sich vorwiegend deutsche Jugendliche, die religiöse Werthaltungen ablehnen. Sie bezeichnen sich überdurchschnittlich oft als Raver, Techno-Fans, Hooligans und Skins bzw. Skinheads. In Münster sind erhöhte Anteile an Haupt- und Sonder- 
schülerinnen und -schülern, in Duisburg 2005 an Realschülerinnen und -schülern auffällig. Im Bereich der Freizeitaktivitäten gibt es vor allem eine Gemeinsamkeit: Die Jugendlichen gehen häufig auf Partys. Bei den Film- und Fernsehvorlieben ist zu konstatieren, dass in Münster oft Horrorfilme geschaut werden und in Duisburg 2005 Kriegsfilme. In Münster 2003 und Duisburg 2005 sind die Alkoholkonsumraten überdurchschnittlich hoch, darüber hinaus sind die Drogenkonsumraten in Duisburg in beiden Erhebungsjahren erhöht. Ein Unterschied zwischen beiden Städten besteht in der Delinquenzbelastung. Während die Delinquenzraten in Duisburg sogar die höchsten aller Typologien sind, bewegen sie sich in Münster nur auf durchschnittlichem Niveau. In diesen Typologien finden sich demnach Belege für das gleichzeitige Auftreten einer Präferenz für Techno und House und erhöhten Alkohol- und Drogenkonsumraten.

Auch die Außenseiter-Typologien finden sich in allen Datensätzen. Sie sind durch die weit verbreitete Ablehnung aller Musikstile mit Ausnahme der Neuen E-Musik gekennzeichnet, in Münster präferieren sogar überdurchschnittlich viele Jugendliche diesen Stil. Auch dieser Typus ist eher „,bildungsfern“: In Münster ist der Anteil der Jugendlichen, die eine Sonderschule und in Duisburg derjenigen, die eine Hauptschule besuchen, deutlich erhöht, in Duisburg 2005 trifft dieser Befund auch auf Gesamtschüler zu. In Duisburg beider Jahre befinden sich überdurchschnittlich viele Türken in diesen Gruppierungen. Im Bereich der Werte fällt eine deutlich erhöhte Zustimmung zu Religion und in Duisburg, damit verbunden, eine Ablehnung von Hedonismus und Technik auf. Die eigene Peer-Group gehört keiner speziellen Gruppe an. In Münster spielt - aufgrund der konstatierten „Bildungsferne“ nicht ganz erwartungsgemäß - ein größerer Anteil oft ein (klassisches) Instrument und geht häufig ins Theater, in Duisburg wird die Freizeit überdurchschnittlich oft mit Familienangehörigen verbracht. Ansonsten ist die überdurchschnittlich verbreitete Ablehnung vieler Freizeitaktivitäten auffällig. Ebenso werden viele Filmgenres (mit Ausnahme der Naturfilme in Duisburg) unterdurchschnittlich häufig geschaut. Diese Ablehnungswerte sind so zahlreich und so weit verbreitet wie in keiner anderen Typologie und sind Grundlage für die Benennung der Typologie. Sie gehen mit niedrigen Alkohol- und sehr niedrigen Drogenkonsumraten einher. Darüber hinaus gehören diese Gruppierungen in Münster und Duisburg zu den unterdurchschnittlich delinquenzbelasteten. In Duisburg sind sie sogar die am wenigsten belasteten überhaupt. Somit lassen sich hier leichte Hinweise für das Zutreffen der Hypothese eines Zusammenhangs zwischen der Vorliebe für Klassik, Jazz und Blues und niedrigen Delinquenzraten ausmachen.

Neben diesen datensatzübergreifend sehr ähnlichen Typologien, treten mehrere unterschiedliche Gruppierungen auf, die durch eine überdurchschnittliche Vorliebe für Rockmusik gekennzeichnet sind. Noch in allen drei Datensätzen ist der Typus der Rocker zu finden. Dieses Profil zeichnet sich durch eine Verbreitung der Zustimmung zu Rock und der Ablehnung der Stile Black Music und Dance (Münster und Duisburg 2003) bzw. aller anderen Stile (Duisburg 2005) aus. Daneben gibt es allerdings in Duisburg 2003 eine weitere und in Duisburg 2005 zwei weitere Typologien mit Affinität zur Rockmusik. Zu dem Musikgeschmack in der Typologie der Rocker scheint eine „rebellische“ unangepasste Werthaltung zu gehören, die in der weit verbreiteten Ablehnung von Tradition und Religion bei Zustimmung zu Deprivation sichtbar wird. In Duisburg 2005 kommt eine häufige Zustimmung zu technikbefürwortenden Wertorientierungen hinzu. In diesen Rocktypologien liegen erhöhte Jungenanteile vor und es wird häufig von einem hohen Sozialstatus berichtet. Dazu passt ein erhöhter Anteil an Jugendlichen, die ein Gymnasium besuchen (in Duisburg 2003 auch eine Realschule), und ein niedriger Migrantenanteil. Auch in diesen Musikgruppierungen bezeichnen die Befragten ihre eigenen Freunde dem Musikgeschmack entsprechend als Punks und Heavy-Metal-Fans. Weiterhin spielen noch Computer-Freaks, Rollenspieler und Antifaschisten eine größere Rolle. In Duisburg werden darüber hinaus noch Skater genannt, Rapper bzw. Hip-Hopper dagegen auffallend selten. In Münster 2003 und Duisburg 2005 finden sich auch erhöhte Anteile an Skins bzw. Skinheads. In allen Datensätzen spielen die Jugendlichen dieser Typologien überdurch- 
schnittlich häufig ein Musikinstrument und machen in einer Band Musik. Sie shoppen selten und verbringen ihre Freizeit selten mit Familienangehörigen, machen selten am eigenen PC Musik und gehen eher selten ins Kino. Sie schauen oft Science-Fiction-Filme und - passend zur schulischen Bildung - auch politische Magazine und Dokumentationen, dagegen selten Unterhaltungsserien, Fernsehshows und Musikvideos. In Duisburg konsumieren sie daneben oft Kriegsfilme, dagegen deutlich seltener Love Stories und Kung-Fu-Filme als der Durchschnitt. In Münster und Duisburg des Jahres 2003 finden sich hier erhöhte Alkohol- und Drogenkonsumraten. Die Delinquenzbelastung ist in allen Erhebungsorten und -jahren durchschnittlich.

Zwei der erwähnten weiteren Rocktypologien sind die der privilegierten Rocker (Duisburg 2005) und unterprivilegierten Rocker (Duisburg 2003). Auch in diesen Gruppierungen liegt eine verbreitete Zustimmung zu dem Musikstil Rock bei Ablehnung von Dance vor, und die Ablehnung von Tradition und Religion entspricht ebenfalls den bereits beschriebenen Rocktypologien. Weiterhin sind die erhöhten Gymnasiastenanteile vergleichbar, jedoch wird in der unterprivilegierten Rocktypologie nicht von einem erhöhten Sozialstatus berichtet, in der privilegierten Rocktypologie dagegen von dem höchsten aller Gruppierungen. Die Jugendlichen beider Typen bezeichnen den eigenen Freundeskreis wiederum als Punks und Heavy-MetalFans, diejenigen des unterprivilegierten Typus nennen hier auch Skater. Im privilegierten Typus werden darüber hinaus noch die Gruppen der Antifaschisten und Brit-Popper überdurchschnittlich oft genannt. Bei ebendiesen privilegierten Jugendlichen wird oft das Spielen eines (klassischen) Musikinstruments, das Musizieren in einer Band und das Singen in einem Chor genannt, Aktivitäten, die in der unterprivilegierten Klasse fehlen. Hier wird überdurchschnittlich oft abgehangen, dagegen selten geshoppt und in der Freizeit werden selten Familienangehörige getroffen. Im privilegierten Typus werden gerne Science-Fiction-Filme sowie politische Dokumentationen und Magazine geschaut, selten dagegen Unterhaltungsserien sowie Horror- und Kung-Fu-Filme. In der unterprivilegierten Rocktypologie fehlt die ,hochkulturelle", politische Komponente aber auch hier werden Unterhaltungsserien und Fernsehshows abgelehnt. In dieser unterprivilegierten Rocktypologie finden sich ein hoher Alkohol- und Drogenkonsum sowie hohe Delinquenzraten, in der privilegierten dagegen in beiden Bereichen nur durchschnittliche Raten.

Der letzte Rocktypus, welcher allerdings nur in Duisburg 2005 existiert, ist derjenige der durchschnittlichen Rocker. Zwar liegt auch hier eine verbreitete Zustimmung zum Musikstil Rock vor, diese ist jedoch niedriger ausgeprägt als in den anderen Rockgruppierungen. Zudem finden sich hier durchschnittliche Zustimmungswerte zu Dance und Black Music. Wie in den anderen Rocktypologien auch, ist der Anteil der Gymnasiasten erhöht und im Bereich der Werthaltungen werden Tradition und Religion abgelehnt. Die Mitglieder der eigenen PeerGroup werden als Punks und Skater beschrieben. Neben diesen Befunden gibt es jedoch aus dieser Klasse keine Auffälligkeiten zu berichten. Sowohl die Freizeitaktivitäten als auch der Film- und Fernsehgeschmack entsprechen dem Durchschnitt. Auch die Raten des Alkoholund Drogenkonsums sowie die Kriminalitätsraten weichen nicht signifikant vom Durchschnitt aller Befragten ab.

Eine Musiktypologie, welche nur in Münster 2003 auftritt, ist die der konformen Durchschnittlichen. Die Jugendlichen dieser Gruppierung haben einen in allen Bereichen durchschnittlichen Musikgeschmack, nur die Zustimmung zur Neuen E-Musik liegt darüber. Der Mädchenanteil ist erhöht, ebenso der Anteil derjenigen, die ein Gymnasium besuchen. Tradition und Hedonismus werden abgelehnt. Im Bereich der Gruppenselbstzuschreibung wird überdurchschnittlich häufig explizit keine Gruppe genannt. Die Jugendlichen aus dieser Klasse schauen gerne Love Stories, dafür selten Horror- und Actionfilme, ein Befund, der sich mit dem erhöhten Mädchenanteil deckt. In dieser Gruppierung der „,braven Mädchen“ liegen die 
niedrigsten Kriminalitätsraten aller Münsteraner Musiktypologien vor. Auch in dieser Gruppe lässt sich somit ein Hinweis auf den Zusammenhang zwischen Klassik, Blues und Jazz und niedrigen Kriminalitätsraten finden.

\section{Fazit}

Zunächst kann mit den vorliegenden Ergebnissen festgehalten werden, dass Jugendliche anhand ihres Musikgeschmacks in stabile und über alle drei Datensätze vergleichbare Gruppen eingeteilt werden können, die schon für sich einen lohnenswerten Einblick in jugendliche Lebenswelten ermöglichen. Daneben kann ein gemeinsames Auftreten sehr breit gefächerter Musikstilpräferenzen, also eines sehr, ,toleranten“ Musikgeschmacks, wie er in den Typologien der Vielseitigen auftritt, und einer höheren Soziallage in allen Datensätzen bestätigt werden.

$\mathrm{Zu}$ der These des Zusammenhangs zwischen Drogen- bzw. Alkoholkonsum und einer Vorliebe für House- und Technomusik (hier zusammengefasst im Musikstil Dance) lässt sich sagen, dass in Münster in beiden Dance-Typologien (Hip-Hopper und Raver sowie Party-Raver) erhöhte Alkoholraten und in Duisburg 2003 in der Party-Raver-Typologie ein höherer Drogenkonsum feststellbar ist. Die Party-Raver aus Duisburg 2005 zeigen erhöhte Alkohol- und Drogenkonsumraten, welche in diesem Datensatz außerdem noch bei den dance-assoziierten Gruppen der Hip-Hopper und Raver zu finden sind. Diese städte- und zeitpunktübergreifenden Auffälligkeiten lassen in der Tat darauf schließen, dass erhöhter Alkohol- und Drogenkonsum mit einer musikalischen Vorliebe für House- und Technomusik einhergeht.

In Bezug auf kriminelles Verhalten ist festzustellen, dass offenkundig eine Verbindung zwischen Rap-Musik und Kriminalität besteht. In Münster sind bei den reinen Hip-Hoppern, für welche eine sehr breite Zustimmung zu Black Music bei Ablehnung aller anderen Stile charakteristisch ist, alle Kriminalitätsraten (etwas unerwartet mit Ausnahme der Gewaltkriminalität) deutlich erhöht. In Duisburg 2003 sind alle Raten bis auf die des Sprayens von Graffiti, in Duisburg 2005 alle Raten bis auf die der Sachbeschädigung signifikant erhöht. Dieser Typus ist somit durchgängig belasteter als die anderen. Daneben scheint es indessen noch einen Zusammenhang zwischen der Vorliebe für Dance und erhöhten Delinquenzraten zu geben: In Münster weist die Gruppe der Hip-Hopper und Raver, in Duisburg 2003 und 2005 weisen die Gruppierungen der Party-Raver erhöhte Delinquenzwerte auf.

Des Weiteren wurde vermutet, dass bei Typologien mit einer Vorliebe zu Neuer E-Musik unterdurchschnittliche Kriminalitätsraten zu erwarten seien. Tatsächlich gehen in Münster beide unterdurchschnittlich kriminalitätsbelasteten Typologien (konforme Durchschnittliche und Außenseiter) mit überdurchschnittlichen Zustimmungswerten zu Neuer E-Musik einher. In Duisburg ist die vermutete Koinzidenz nicht so eindeutig nachzuweisen. Es existieren zwar ebenfalls unterdurchschnittlich belastete Außenseiter-Typologien mit einer Ablehnung aller Stile bis auf die der Neuen E-Musik. Dieser Stil wird hier jedoch nur durchschnittlich weit verbreitet präferiert. In Duisburg 2003 ist bei einem zweiten Typus, den vielseitigen Mädchen, die Gesamtdelinquenz ebenfalls unterdurchschnittlich ausgeprägt und die Neue E-Musik überdurchschnittlich weit verbreitet beliebt. Diese Vorliebe ist allerdings kombiniert mit weiteren Vorlieben für die Musikstile Rock und Dance.

Prägnant formuliert kann man im Bereich der Jugendkriminalität also davon sprechen, dass die Musiktypologien der reinen Hip-Hopper über- und die der Außenseiter unterdurchschnittlich kriminalitätsbelastet sind. In Duisburg beider Jahre trifft eine besondere Delinquenzbelastung darüber hinaus noch auf die Gruppierungen der Party-Raver zu. Es existiert also ein durchaus stabiler Kern an höher und niedriger belasteten Musiktypologietypen, der in allen Datensätzen zu beobachten ist. Allerdings muss an dieser Stelle darauf hingewiesen werden, dass mit der ausgeführten Vorgehensweise keine Aussagen über Kausalzusammenhänge ge- 
macht werden können. Die Musiktypologien können zwar als Indikatoren für Jugendszenen gelten, in denen abweichendes Verhalten häufiger oder seltener vorkommt als in anderen - um deviantes oder gar delinquentes Verhalten erklären zu können, müssen jedoch viele weitere Ursachen herangezogen werden. Ein monokausaler Erklärungsversuch mit Musikgeschmack als Ursache für abweichendes Verhalten kann nur zu kurz greifen. In diesem Zusammenhang ist auch zu konstatieren, dass die vorgestellten Ergebnisse im Hinblick auf die Präferenzen der einzelnen Musikstile nicht eindeutig sind: Erhöhte Konsumraten scheinen neben dem Musikstil Dance auch mit Rock-Musik in Verbindung zu stehen und es existieren durchaus Typologien, in denen zwar eine Präferenz für Neue E-Musik vorherrscht, die aber keine (hypothesenkonforme) unterdurchschnittliche Kriminalitätsbelastung aufweisen. Ebenso gibt es Gruppierungen, in denen zwar eine Vorliebe für Black Musik verbreitet ist, aber keine erhöhten Delinquenzraten auftreten, und in einigen delinquenzbelasteten Typologien wird Black Music abgelehnt.

Basierend auf den hier vorgestellten Ergebnissen erscheint es für die weitere Forschung lohnenswert, der Frage nach den Erklärungen für abweichendes Verhalten in den einzelnen Typologien nachzugehen. Welche typologiespezifischen Ursachenbündel lassen sich ausmachen und wie stellen sich Gruppenwechsel von Jugendlichen gerade im Hinblick auf kriminelles Verhalten dar? Die Daten des vorgestellten Projektes bieten aufgrund der jährlichen Fortschreibung die Möglichkeit, mit Hilfe der zeitlich an diese Analyse anschließenden Datensätze die Ergebnisse laufend zu validieren. Einen besonders vielversprechenden Untersuchungsansatz bieten darüber hinaus die mittlerweile zur Verfügung stehenden Paneldaten. Mit ihnen können die Entwicklung der Typologientypen über die Zeit und die Verläufe der Gruppenzugehörigkeit der Jugendlichen nachgezeichnet werden. Gehören junge Menschen überwiegend denselben Musiktypologien an oder sind hier unter Berücksichtigung eines sich wandelnden Musikgeschmacks dynamische Verläufe zu entdecken, die mit unterschiedlichen Phasen abweichenden Verhaltens korrespondieren?

\section{Literatur}

Anderson, Craig A. / Nicholas L. Carnagey / Janie Eubanks (2003): Exposure to Violent Media: The Effect of Songs With Violent Lyrics on Aggressive Thoughts and Feelings, in: Journal of Personality and Social Psychology 84, S. 960-971.

Andreß, Hans-Jürgen / Jacques A. Hagenaars / Steffen-M. Kühnel (1997): Analyse von Tabellen und kategorialen Daten. Log-lineare Modelle, latente Klassenanalyse, logistische Regression und GSKAnsatz, Berlin, Heidelberg et al.

Baacke, Dieter (1997): Die Welt der Musik und die Jugend, in: Dieter Baacke (Hrsg.), Handbuch Jugend und Musik, Opladen, S. 9-26.

Baacke, Dieter (2004): Jugend und Jugendkulturen. Darstellung und Deutung. 4. Auflage, Weinheim München.

Bacher, Johann / Knut Wenzig / Melanie Vogler (2004): SPSS TwoStep Cluster - A First Evaluation, 2. Auflage, Arbeits- und Diskussionspapiere des Lehrstuhls für Soziologie \& Empirische Sozialforschung der Friedrich-Alexander-Universität Nürnberg-Erlangen, Bd. 2004-2 (Arbeits- und Diskussionspapiere des Lehrstuhls für Soziologie \& Empirische Sozialforschung der Friedrich-Alexander-Universität Nürnberg-Erlangen. Bd. 2004-2), Nürnberg.

Bacher, Johann / Andreas Pöge / Knut Wenzig (2010): Clusteranalyse. Anwendungsorientierte Einführung in Klassifizierungsverfahren, 3. Auflage, München.

Baier, Dirk / Michael Windzio (2008): Zur Entwicklung der Jugendgewalt seit 1998 in den Städten München, Stuttgart, Hannover und Schwäbisch Gmünd, in Karl-Siegbert Rehberg (Hrsg.), Die Natur der Gesellschaft. Verhandlungen des 33. Kongresses der Deutschen Gesellschaft für Soziologie in Kassel 2006, Frankfurt / Main, CD-ROM, S. 4560-4575. 
Behne, Klaus-Ernst (2007): Aspekte einer Sozialpsychologie des Musikgeschmacks, in: Helga de la MotteHaber / Hans Neuhoff (Hrsg.), Musiksoziologie, Laaber, S. 418-427.

Boers, Klaus / Andreas Pöge (2003): Wertorientierungen und Jugenddelinquenz, in: Siegfried Lamnek / Manuela Boatca (Hrsg.), Geschlecht, Gewalt, Gesellschaft, Opladen, S. 246-269.

Boers, Klaus / Jost Reinecke (Hrsg.) (2007): Delinquenz im Jugendalter. Erkenntnisse einer Münsteraner Längsschnittstudie, Münster - New York et al.

Boers, Klaus / Jost Reinecke / Christina Bentrup / Kristina Kanz / Susann Kunadt / Luca Mariotti / Andreas Pöge / Daniela Pollich / Daniel Seddig / Christian Walburg / Jochen Wittenberg (2010): Jugendkriminalität - Altersverlauf und Erklärungszusammenhänge, in: Forum Kriminalprävention 4, S. 48-56

Boers, Klaus / Christian Walburg / Jost Reinecke (2006): Jugendkriminalität - Keine Zunahme im Dunkelfeld, kaum Unterschiede zwischen Einheimischen und Migranten. Befunde aus Duisburger und Münsteraner Längsschnittstudien, in: Monatsschrift für Kriminologie und Strafrechtsreform 89, S. 63-87.

Boers, Klaus / Christian Walburg (2007): Verbreitung und Entwicklung delinquenten und abweichenden Verhaltens unter Jugendlichen, in: Klaus Boers / Jost Reinecke (Hrsg.), Delinquenz im Jugendalter. Erkenntnisse einer Münsteraner Längsschnittstudie, Münster - New York et al., S. 79-96.

Bortz, Jürgen (1999): Statistik für Sozialwissenschaftler, 5. Auflage, Berlin - Heidelberg et al.

Bourdieu, Pierre (1987): Die feinen Unterschiede. Kritik der gesellschaftlichen Urteilskraft, 4. Auflage (La distinction. Critique sociale du jugement. Paris: Les éditions de minuit), Frankfurt / Main.

Brondies, Marc (2004): Methodendokumentation der kriminologischen Schülerbefragung in Duisburg 2003, Schriftenreihe „Jugendkriminalität in der modernen Stadt - Methoden“ Nr. 6, Münster - Trier.

Bühl, Achim / Peter Zöfel (2005): SPSS 12. Einführung in die moderne Datenanalyse unter Windows, 9. Auflage, München.

Bundeskriminalamt (Hrsg.) (2005): Polizeiliche Kriminalstatistik. Bundesrepublik Deutschland. Berichtsjahr 2004, Wiesbaden.

Bundeskriminalamt (Hrsg.) (2009): Polizeiliche Kriminalstatistik Bundesrepublik Deutschland. Berichtsjahr 2008, Wiesbaden.

Bundeskriminalamt (Hrsg.) (2010): Polizeiliche Kriminalstatistik Bundesrepublik Deutschland. Berichtsjahr 2009, Wiesbaden.

Bundesministerium des Innern und Bundesministerium der Justiz (Hrsg.) (2006): Zweiter Periodischer Sicherheitsbericht, Berlin.

Bundesverband der Unfallkassen (Hrsg.) (2005): Gewalt an Schulen. Ein empirischer Beitrag zum gewaltverursachten Verletzungsgeschehen an Schulen in Deutschland 1993-2003, München, abrubar unter http://www.dguv.de/inhalt/zahlen/documents/Gewalt_an_Schulen.pdf, letztes Abrufdatum: 2.7.2011.

Bund-Länder-AG „Entwicklung der Gewaltkriminalität junger Menschen mit einem Schwerpunkt auf städtischen Ballungsräumen“" (2007): Bericht zur IMK-Herbstsitzung 2007 (Berichtsstand 16. November 2007), abrufbar unter: http://www.berlin.de/imperia/md/content/seninn/imk2007/beschluesse/ imk_185_bericht_top16.pdf?start\&ts=1197380935\&file=imk_185_bericht_top16.pdf, letztes Abrufdatum 2.7.2011.

von Davier, Matthias (1997): Methoden zur Prüfung probabilistischer Testmodelle, Kiel.

Deutsche Shell (Hrsg.) (2000): Jugend 2000, 13. Shell Jugendstudie, Bd. 1, Opladen.

Deutsche Shell (Hrsg.) (2006): Jugend 2006, 15. Shell Jugendstudie, Frankfurt / Main.

Deutsche Shell (Hrsg.) (2010): Jugend 2010, 16. Shell Jugendstudie, Frankfurt / Main.

Dünkel, Frieder / Dirk Gebauer / Bernd Geng (2007): Gewalterfahrungen, gesellschaftliche Orientierungen und Risikofaktoren von Jugendlichen in der Universitäts- und Hansestadt Greifswald 1998 - 2002 2006. Erste zentrale Ergebnisse einer Langzeitstudie zur Lebenssituation und Delinquenz von Jugendlichen in der Universitäts- und Hansestadt Greifswald, www.rsf.uni-greifswald.de/fileadmin/media- 
pool/lehrstuehle/duenkel/Schuelerbefragung_HGW_1998_2002_2006.pdf, letztes Abrufdatum: 14.5.2011.

Dünkel, Frieder / Dirk Gebauer / Bernd Geng et al. (2005): Young migrants and members of ethnic minorities as victims and offenders of violent crimes: an international comparative survey in the region of the Baltic Sea, in: Nicolas Queloz / Repond Frédérique Bütikofer / Delphine Pittet et al. (Hrsg.), Délinquance des jeunes et justice des mineurs. Les défis des migrations et de la pluralité ethnique. Youth Crime and Juvenile Justice. The challenge of migration and ethnic diversity, Berne - Bruxelles, S. $185-220$.

Farin, Klaus (2001): generation-kick.de: Jugendsubkulturen heute, München.

Ferchhoff, Wilfried / Uwe Sander / Ralf Vollbrecht (Hrsg.) (1995): Jugendkulturen - Faszination und Ambivalenz. Einblicke in jugendliche Lebenswelten, Opladen.

Fischer, Peter / Greitemeyer, Tobias (2006): Music and Aggression: The Impact of Sexual-Aggressive Song Lyrics on Aggression-Related Thoughts, Emotions, and Behavior Towards the Same and the Opposite Sex, in: Personality and Social Psychology Bulletin 32, S. 1165-1176.

Forsyth, Alasdair J. M. / Marina Barnard / Neil P. McKeganey (1997): Musical preference as an indicator of adolescent drug use, in: Addiction 92, S. 1317-1325.

Fuchs, Marek / Siegfried Lamnek / Jens Luedtke et al. (2005): Gewalt an Schulen. 1994 - 1999 - 2004, Wiesbaden.

Gardstrom, Susan C. (1999): Music Exposure and Criminal Behavior: Perceptions of Juvenile Offenders, in: Journal of Music Therapy 36, S. 207-222.

Gembris, Heiner (2005): Musikalische Präferenzen, in: Rolf Oerter / Thomas H. Stoffer (Hrsg.), Enzyklopädie der Psychologie. Spezielle Musiksoziologie. Musiksoziologie Nr. 2, Göttingen - Bern et al., S. 279-342.

Greve, Martin (2007): Musik nach Migration: Türkische Musik in Deutschland, in: Helga de la MotteHaber / Hans Neuhoff (Hrsg.), Musiksoziologie, Laaber, S. 518-534.

Heinz, Wolfgang (2006): Kriminelle Jugendliche - gefährlich oder gefährdet?, Konstanz.

Hitzler, Ronald / Thomas Bucher / Arne Niederbacher (2005): Leben in Szenen. Formen jugendlicher Vergemeinschaftung heute, 2. Auflage, Wiesbaden.

Johnson, James D. / Mike S. Adams / Leslie et al. Ashburn (1995): Differential Gender Effects of Exposure to Rap Music on African American Adolescents' Acceptance of Teen Dating Violence, in: Sex Roles 33, S. 597-605.

Johnson, James D. / Lee Anderson Jackson / Leslie Gatto (1995): Violent Attitudes and Deferred Academic Aspirations: Deleterious Effects of Exposure to Rap Music, in: Basic and Applied Social Psychology 16 , S. 27-41.

Kleinen, Günter (2007): Musikalische Lebenswelten, in: Helga de la Motte-Haber und Hans Neuhoff (Hrsg.), Musiksoziologie, Laaber, S. 438-455.

Köllisch, Tilman / Dietrich Oberwittler (2004): Wie ehrlich berichten männliche Jugendliche über ihr delinquentes Verhalten? Ergebnisse einer externen Validierung, in: Kölner Zeitschrift für Soziologie und Sozialpsychologie 56, S. 708-735.

Krauth, Joachim / Gustav A. Lienert (1973): Die Konfigurationsfrequenzanalyse (KFA) und ihre Anwendung in Psychologie und Medizin. Ein multivariates nichtparametrisches Verfahren zur Aufdeckung von Typen und Syndromen, Freiburg / Breisgau - München.

Kunadt, Susann (2006): Methodendokumentation der kriminologischen Schülerbefragung in Duisburg 2005, Schriftenreihe „Jugendkriminalität in der modernen Stadt - Methoden“ Nr. 11, Münster - Bielefeld.

Lacourse, Eric / Michel Claes / Martine Villeneuve (2001): Heavy Metal Music and Adolescent Suicidal Risk, in: Journal of Youth and Adolescence 33, S. 321-332. 
Lautsch, Erwin / Stefan von Weber (1995): Methoden und Anwendungen der Konfigurationsfrequenzanalyse (KFA), Weinheim.

Lazarsfeld, Paul F. (1950): The Logical and Mathematical Foundations of Latent Structure Analysis, in: Samuel Andrew Stouffler / Louis Guttman / Edward Allen Suchman et al. (Hrsg.), Studies in Social Psychology in World War II. Bd. IV: Measurement and Prediciton, Princeton / NJ, S. 362-412.

Lazarsfeld, Paul F. / Neil W. Henry (1968): Latent structure analysis, Boston.

Lösel, Friedrich / Thomas Bliesener / Mechthild Averbeck (1999): Hat die Delinquenz von Schülern zugenommen? Ein Vergleich im Dunkelfeld nach 22 Jahren, in: Mechthild Schäfer / Dieter Frey (Hrsg.), Aggression und Gewalt unter Kindern und Jugendlichen, Göttingen - Bern et al., S. 65-89.

Mansel, Jürgen / Klaus Hurrelmann (1998): Aggressives und delinquentes Verhalten Jugendlicher im Zeitvergleich. Befunde aus ,Dunkelfeldforschungen“ aus den Jahren 1988, 1990 und 1996, in: Kölner Zeitschrift für Soziologie und Sozialpsychologie 50, S. 78-109.

Martin, Graham / Michael M. B. Clarke / Colby B. A. Pearce (1993): Adolescent Suicide: Music Preference as an Indicator of Vulnerability, in: Journal of the American Academy of Child \& Adolescent Psychiatry 32, S. 530-535.

Miranda, Dave / Michael Claes (2004): Rap Music Genres and Deviant Behaviors in French-Canadian Adolescents, in: Journal of Youth and Adolescence 33, S. 113-122.

Neuhoff, Hans (2007): Die Konzertpublika der deutschen Gegenwartskultur. Empirische Publikumsforschung in der Musiksoziologie, in: Helga de la Motte-Haber / Hans Neuhoff (Hrsg.), Musiksoziologie, Laaber, S. 473-509.

Neuhoff, Hans / Helga de la Motte-Haber (2007): Musikalische Sozialisation, in: Helga de la Motte-Haber / Hans Neuhoff (Hrsg.), Musiksoziologie, Laaber, S. 389-417.

Pederson, Willy / Anders Skrondal (1999): Ecstacy and new patterns of drug use: A normal population study, in: Addiction 94, S. 1695-1706.

Peterson, Richard / Roger Kern (1996): Changing Highbrow Taste: From Snob to Omnivore, in: American Sociological Review 61, S. 900-907.

Pfeiffer, Christian / Ingo Delzer / Dirk et al. Enzmann (1998): Ausgrenzung, Gewalt und Kriminalität im Leben junger Menschen. Kinder und Jugendliche als Opfer und Täter. (Sonderdruck zum 24. Deutschen Jugendgerichtstag vom 18.-22. September 1998 in Hamburg, Hannover 1998), Hannover.

Pfeiffer, Christian / Thomas Ohlemacher (1995): Anstieg der (Gewalt-)Kriminalität und der Armut junger Menschen. Gibt es einen Zusammenhang?, in: Siegfried Lamnek (Hrsg.), Jugend und Gewalt. Devianz und Kriminalität in Ost und West, Opladen, S. 259-277.

Pöge, Andreas (2002): Der Zusammenhang von Lebensmilieu, Einstellungen und deviantem Verhalten bei Jugendlichen, Münster (unveröffentlichte Magisterarbeit).

Pöge, Andreas (2007 a): Soziale Jugendmilieus und Delinquenz, in: Klaus Boers / Jost Reinecke (Hrsg.), Delinquenz im Jugendalter. Erkenntnisse einer Münsteraner Längsschnittstudie, Münster - New York et al., S. 201-240.

Pöge, Andreas (2007 b): Soziale Milieus und Kriminalität im Jugendalter. Eine Untersuchung von Werteund Musiktypologien in Münster und Duisburg, Münster - New York et al.

Pöge, Andreas / Jochen Wittenberg (2007): Untersuchungsdesign und Stichproben der Münsteraner Schülerbefragungen, in: Klaus Boers / Jost Reinecke (Hrsg.), Delinquenz im Jugendalter. Erkenntnisse einer Münsteraner Längsschnittstudie, Münster - New York et al., S. 57-74.

Primack, Brian A. / Madeline A. Dalton / Mary V. Carroll et al. (2008): Content Analysis of Tobacco, Alcohol, and Other Drugs in Popular Music, in: Archives of Pediatrics \& Adolescent Medicine 162, S. 169-175.

Raithel, Jürgen (2004): Jugendliches Risikoverhalten. Eine Einführung, Wiesbaden.

Roe, Keith, 1992: Different Destinies - Different Melodies: School Achievement, Anticipated Status and Adolescents' Tastes in Music, in: European Journal of Communication 7, S. 335-357. 
Roe, Keith (1995): Adolescents' Use of Socially Disvalued Media: Towards a Theory of Media Delinquency, in: Journal of Youth and Adolescence 24, S. 617-631.

Rössler, Patrick / Nadeschda Scharfenberg (2004): Wer spielt die Musik? Kommunikationsnetzwerke und Meinungsführerschaft unter Jugendlichen - eine Pilotstudie zu Musikthemen, in: Kölner Zeitschrift für Soziologie und Sozialpsychologie 56, S. 490-519.

Schmidbauer, Michael / Paul Löhr (1999): See me, feel me, touch me. Das Publikum von MTV Europe und VIVA, in: Klaus Neumann-Braun (Hrsg.), Viva MTV! Popmusik im Fernsehen. Frankfurt / Main, S. 325-349.

Schulze, Gerhard (1992): Die Erlebnisgesellschaft: Kultursoziologie der Gegenwart, Frankfurt / Main.

Schwarz, Gideon (1978): Estimating the Dimension of a Model, in: Annals of Statistics 6, S. 461-464.

Spellerberg, Anette (1996): Soziale Differenzierung durch Lebensstile. Eine empirische Untersuchung zur Lebensqualität in West- und Ostdeutschland, Berlin.

Stadt Münster (Hrsg.) (2001): Jugendumfrage 2001 (Beiträge zur Statistik 83, Amt für Stadt- und Regionalentwicklung, Statistik), Münster.

Steffen, Wiebke (2007): Jugendkriminalität und ihre Verhinderung zwischen Wahrnehmung und empirischen Befunden. Gutachten zum 12. Deutschen Präventionstag am 18. und 19. Juni 2007 in Wiesbaden, abrufbar unter: http://www.praeventionstag.de/html/GetDokumentation.cms?XID=227, letztes Abrufdatum: 11.7.2011.

Tillmann, Klaus-Jürgen / Birgit Holler-Nowitzki / Heinz Günter Holtappels et al. (1999): Schülergewalt als Schulproblem. Verursachende Bedingungen, Erscheinungsformen und pädagogische Handlungsperspektiven, Weinheim - München.

Took, Kevin J. / Weiss, David S. (1994): The Relationship Between Heavy Metal and Rap Music and Adolescent Turmoil: Real or Artifact?, in: Adolescence 29, S. 613-621.

Vermunt, Jeroen K. / Magidson, Jay (2005 a): Latent GOLD 4.0 User's Guide, Belmont / MA.

Vermunt, Jeroen K. / Jay Magidson (2005 b): Technical Guide for Latent GOLD 4.0: Basic and Advanced, Belmont / MA.

Walter, Michael (1996): Kriminalpolitik mit der polizeilichen Kriminalstatistik? Artikulation eines Unbehagens über den derzeitigen Kurs der Deutschen Jugendgerichtsvereinigung, in: DVJJ-Journal 7, S. 209-215.

Wilmers, Nicola / Dirk Enzmann / Dagmar Schaefer et al. (2002): Jugendliche in Deutschland zur Jahrtausendwende: Gefährlich oder gefährdet? Ergebnisse wiederholter, repräsentativer Dunkelfelduntersuchungen zu Gewalt und Kriminalität im Leben junger Menschen 1998-2000. Interdisziplinäre Beiträge zur kriminologischen Forschung Bd. 23, Baden-Baden.

Wittenberg, Jochen (2004): Methodendokumentation der kriminologischen Schülerbefragung in Münster 2003. Schriftenreihe „Jugendkriminalität in der modernen Stadt - Methoden“ Nr. 7, Münster - Trier.

Dr. Andreas Pöge Universität Bielefeld Fakultät für Soziologie Postfach 100131 33501 Bielefeld andreas.poege@uni-bielefeld.de 